\title{
UNSTEADY AERODYNAMICS OF STATIONARY ELLIPTIC CYLINDERS IN SUBCRITICAL FLOW
}

\author{
by \\ E. WILAND \\ B.Sc: (Hons.), The University of Strathclyde, 1965
}

A THESIS SUBMITTED IN PARTIAL FULFILLMENT OF THE REQUIREMENTS FOR THE DEGREE OF

$$
\text { M.A.Sc. }
$$

in the Department of

Mechanical Engineering

We accept this thesis as conforming to required standard

THE UNIVERSITYY OF BRITISH COLUMBIA

Apri1, 1968 
In presenting this thesis in partial fulfilment of the requirements for an advanced degree at the University of British Columbia, I agree that the Library shall make it freely available for reference and study. I further agree that permission for extensive copying of this thesis for scholarly purposes may be granted by the Head of my Department or by his representatives. It is understood that copying or publication of this thes is for financial gain shall not be allowed without my written permission.

E. Wiland

Department of Mechanical Engineering

The University of British Columbia Vancouver 8, B.C.

Date April 23-1968 


\section{ABSTRACT}

The aerodynamics of a set of two-dimensional elliptic cylinders with eccentricity of 0.8 and 0.6 is studied experimentally during the organised wake condition. The dynamic calibration of the transducer used for measurement of fluctuating pressures is described in detail. The data on Strouhal number, unsteady pressures and wake geometry are presented as a function of angle of attack during static condition of the mode is. The effect of Reynolds number on the fluctuating pressure is also examined. The results indicate dependence of the unsteady forces on Reynolds number at zero angle of attack. Basing the Strouhal number on projected width appears to reduce its dependence on the angle of attack of the models. The existence of a large phase angle between the fluctuating pressures is of interest. The wake geometry study indicates a gradual reduction in the ratio of the lateral to the longitudinal spacing with increase in angle of attack. 


\section{TABLE OF CONTENTS} Mode 15 and Supporting System............. 6

Bibliography. 65

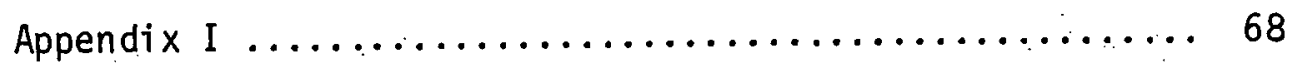

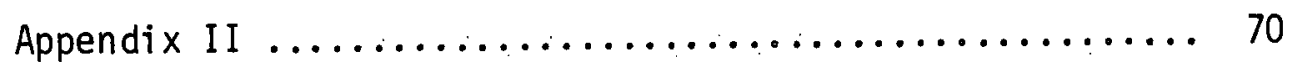




\section{LIST OF TABLES}

Table

Page

$1 \quad$ Mode 1 data............................. 6

2 Effect of phase shift on fluctuating lift coefficient... 54

3. Spacing of vortices in fully developed wake, $\mathrm{Nr}=70,00058$ 


\section{LIST OF FIGURES}

Figure

Constructional details of models.

Numbering of pressure taps.

Wind tunnel outline.

10

Wind tunnel test section with model.

Schematic of Barocel pressure transducer.

Block diagram of pressure generator and set-up for

comparison of dynamical response

Response of calibration system to different input wave

forms

Pressure attenuation as a function of tube length and

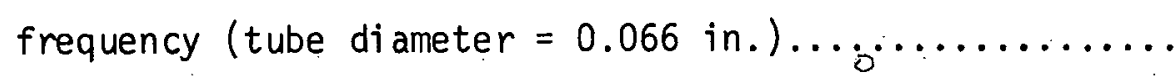

Calibration plots for Barocel pressure transducer. 20

Block diagram of the calibration apparatus.

Calibration apparatus

Calibration plots for Barocel pressure transducer with damping bottle.

Comparison between calibration curves for Barocel pressure transducer with and without damping bottle.

Block diagram of the fluctuating pressure measuring 
Figure

Page

Block diagram of the phase measuring system...........

Instrumentation set-up during a typical test run 35

Traversing gear with probe during wake measurements......

Variation of Strouhal frequency with Reynolds number..... 39

Variation of Strouhal number with angle of attack........

Variation of fluctuating pressure coefficient with

Reynolds number, $e=0.6$.

Variation of fluctuating pressure coefficient with

Reynolds number, $e=0.8$.

Distribution of mean and fluctuating pressure coefficients,

$a=0$

Distribution of mean and fluctuating pressure coefficients, $\alpha=30^{\circ}$

Distribution of mean and fluctuating pressure coefficients, $\alpha=60^{\circ}$. . .

Distribution of mean and fluctuating pressure coefficients, $\alpha=90^{\circ}$

Distribution of mean and fluctuating pressure coefficients

on the surface of a circular cylinder $(e=0)$.

Variation of lift and maximum fluctuating pressure

coefficients with angle of attack...

Amplitude modulation of the pressure signals on the

surface of the model, $e=0.6$

Amplitude modulation of the pressure signals on the surface of the model, $e=0.8$

Phase shift between pressure signals on the surface of

the model, $e=0.6, \alpha=0,90^{\circ} \ldots \ldots \ldots \ldots \ldots \ldots \ldots \ldots$. 
Figure

34. Phase shift between pressure signals on the

surface of the model, $\mathrm{e}=0.8 ; \alpha=0,90^{\circ}$ and

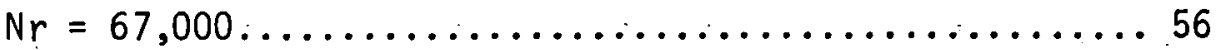

35 Representative results of wake measurements......... 59

(a) Typical wake traverse

(b) Typical amplitude decay in wake

36

Variation of drag and moment coefficients with

angle of attack......................... 61 
ACKNOWLEDGEMENT

The author wishes to express his appreciation for the helpful supervision and advice by Dr. V.J: Modi.

Thanks are also due to the Department of Mechanical Engineering for use of their facilities and to technicians of the department for their valuable assistance.

Financial support was received from the National Research Council of Canada, Grant A-2181. 


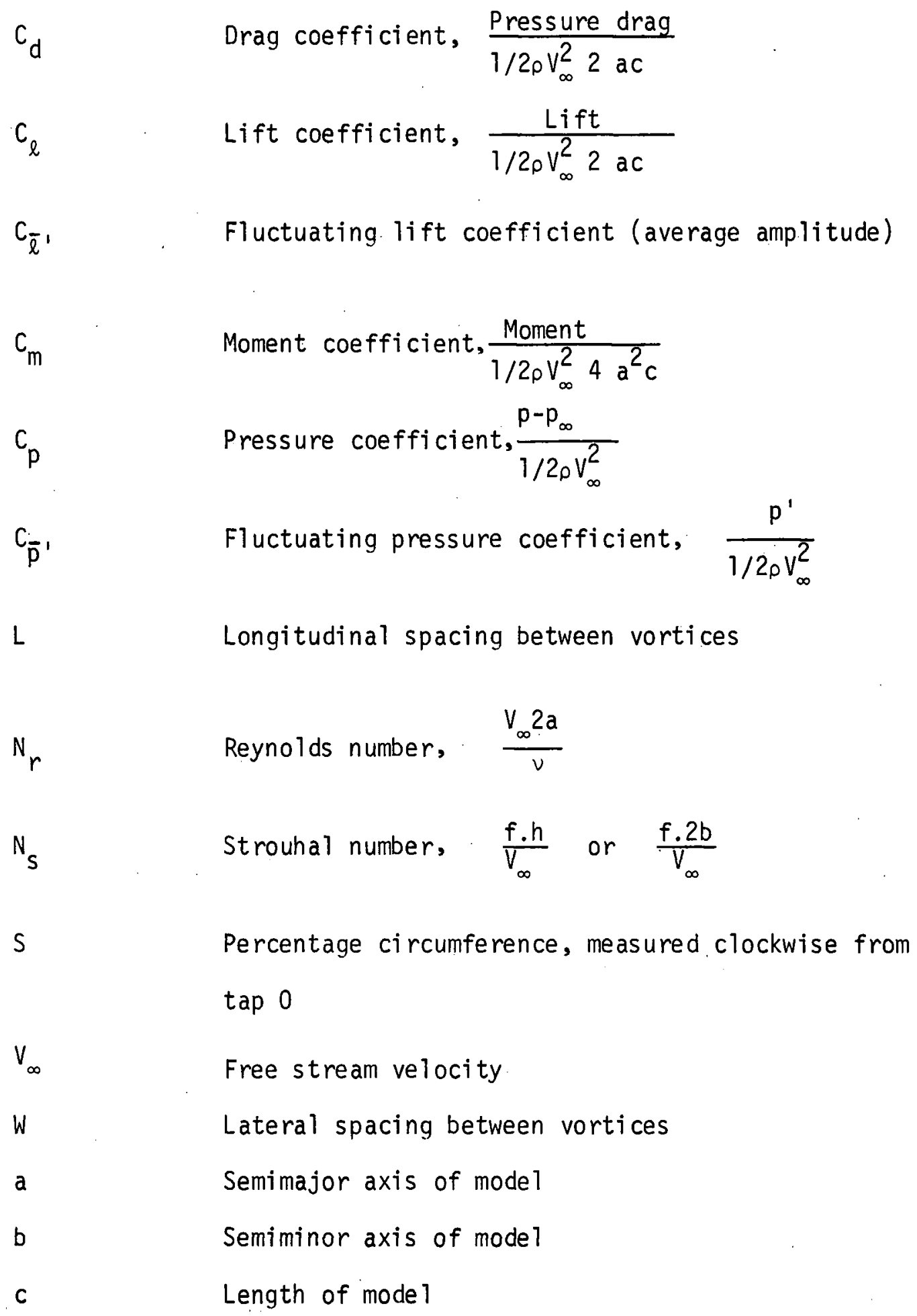


e
Eccentricity, $\sqrt{1-\frac{b^{2}}{a^{2}}}$

Frequency of vortex formation, Strouhal frequency

Projected height of mode $1,2 \sqrt{a^{2} \sin ^{2} \alpha+b^{2} \cos ^{2} \alpha}$

Distance downstream from centre of mode 1

Mean pressure

Fluctuating pressure (average amplitude) about mean

Free stream static pressure

Transverse distance from centre of mode 1

Angle of attack

Air density

Air kinematic viscosity 


\section{INTRODUCT ION}

It is well known that under certain conditions elastically mounted bluff bodies, when exposed to a fluid stream, may exhibit self-induced oscillations. The vibration of smoke stacks, transmission lines, periscopes, aircraft wings, bridges, launch vehicles, etc., has been of interest to engineers. The vibration of a structure is undesirable for many reasons, one of which is the danger of its collapse due to fatigue of the material. In general, the nature of the forcing function, wake geometry, and Strouhal number form three important parameters in an aeroelastic instability study. The determination of the corresponding information for a set of stationary elliptic cylinders in the Reynolds number range of $3 \times 10^{4}-10^{5}$ forms the subject of this presentation.

In general, the elastically supported bluff bodies exhibit two distinct forms of aerodynamically induced vibration transverse to the flow direction. The first type, commonly known as vortex resonance, refers to the condition where the periodicity of the organized vortex shedding coincides with the natural frequency of the system. The second form of oscillation arises due to negative slope in the transverse force versus angle of attack diagram. This causes the body to be unstable under transverse disturbances resulting in oscillation that grows in amplitude until the energy extracted from the fluid stream balances that dissipated through various forms of damping. Strouha ${ }^{1}$ was the first to correlate the periodic vortex shedding with the diameter of a circular cylinder and fluid velocity. 
This was followed by the classical study of wake geometry and stability by Von Karman? ${ }^{2}$. Ever since, academic and practical interest in the vortex shedding phenomenon has resulted in many theoretical and experimental investigations, e.g., by Roshko, Kovasnay, Tritton, Birkhoff, Humphreys, Schaeffer, Eskinazi, and others. Marris ${ }^{3}$ has presented an excellent review of this literature. More recently, Grove et $a 1^{4}$ as well as Bishop and Hassan ${ }^{5}$ measured fluctuating forces on a stationary circular cylinder over a range of Reynolds number. The corrresponding preliminary results for square and rectangular cylinders were presented by Modi and Heine ${ }^{6}$.

In contrast to this, the unsteady pressure variations and wake structure associated with the oscillating two dimensional bluff cylinders have hardly been investigated. The study of Bishop and Hassan ${ }^{7}$ showed that the vortex frequency, over a range of circuiar cylinder frequencies, is controlled; while Fergus on and Parkinson 8 measured fluctuating pressure and wake pattern related to the cylinder executing vortex induced oscillations.

A study of the related but somewhat modified problem involving calculation of the boundary layer and its separation over an infinite, yawed cylinder was carried out by Chi ${ }^{9}$ who also measured its Strouhal number using a flume. It was suggested that the vortex shedding of a yawed cylinder depends only on the cross component of the local velocity.

Among the numerous papers written on the aspects of fluid mechanics and dynamics of bluff bodies, only a few are concerned with the actual measurements of unsteady pressures. McGregor ${ }^{10}$ and Gerrard 11 measured fluctuating forces on a circular cylinder using a condenser 
microphone system built into the mode1. The pressure distribution was obtained by turning the cylinder. Pendergast ${ }^{12}$ obtained spanwise pressure correlation results, for a stationary cylinder, using a modified form of McGregor's apparatus. Keefe ${ }^{13}$ carried out fluctuating force measurements with the help of a carefully designed straingauge transducer which also acted as a test model. Molyneux ${ }^{14}$ has also described a low frequency strain-gauge type transducer mounted inside the model to measure pressures on oscillating wings. Heine ${ }^{15}$ and Ferguson ${ }^{16}$ designed pressure transducers, using a piezolectric crystal and light sensitive resistances respectively, which were located externally and connected to the pressure taps on the model by a series of polyethylene tubings. The devices exhibited several undesirable features. The former has a marginal sensitivity while the latter, though sensitive, was substantially affected by the ambient temperature and humidity.

The available information concerning bluff body interaction with the separated flow of stable vortex street type is not limited to the cylinders of circular cross-section. Investigations with square, rectangular, triangular and hexagonal cylinders, structural $\mathrm{H}$ and angle sections, as well as several irreqular geometries are reported. But it must be emphasized that the bulk of the literature is indeed devoted to the circular geometry. This point is well emphasized by the fact that the previous work on elliptical cylinders seems to be limited to rather preliminary unsteady pressure measurements by Heine ${ }^{15}$ and the Strouhal number study by Schramm ${ }^{17}$. 
The aeroelastic instability of bluff bodies has been under investigation in this department since 1958. The review of the progress made has been reported in two survey papers 18,19 . The investigation described here forms the part of this continuing programme and intends to study, experimentally, the effect of eccentricity of the cylindrical bluff body on the fundamental parameters listed before. 
2. PURPOSE AND SCOPE OF THE INVESTIGATION

As pointed out before, considerable information about the vortex excited motion of a circular cylinder is reported in literature. The long range aim of this project is to obtain corresponding information for two dimensional cylinders, of intermediate cross-sections, obtained by systematically varying the eccentricity from zero (circular cylinder) to infinity (flat plate).

In general the accuracy of the measured data depends on the capability of the instrumentation and the accuracy of its calibration. This being the case, the project studies in detail the dynamic calibration of a transducer used to measure the magnitude, phase relation, and frequency of the acoustic level pressure variations.

The main aim of the project is to study the aerodynamics of two dimensional, elliptic cylindrical models, of eccentricities 0.6 and 0.8 , under the condition conducive to vortex excited oscillations. The thesis presents experimental results on:

(i) the variation of Strouhal number with Reynolds number;

(ii) the mean and fluctuating static pressure distribution;

(iii) wake geometry

as a function of angle of attack during static condition of the model. In most cases the Reynolds number range is confined to $3 \times 10^{4}-10^{5}$. Since the influence of the wind tunnel walls on the measured parameters is not well established, the results presented are uncorrected for that effect (Appendix I). 
3. MODELS AND SUPPORTING SYSTEM

Two elliptic cylindrical models, 27 inches long, were designed to span the wind-tunnel cross-section thus approximating the twodimensional flow condition. The constructional details are shown in Figure 1 and the physical parameters are listed below:

\begin{tabular}{|c|c|c|c|c|c|c|}
\hline Mode 1 & $\mathrm{e}$ & $\mathrm{a} / \mathrm{b}$ & Material & $\begin{array}{l}\text { Weight, } \\
\text { Lb. }\end{array}$ & $\begin{array}{l}\text { Number of } \\
\text { Bulkheads }\end{array}$ & $\begin{array}{l}\text { Skin Thickness, } \\
\text { In. }\end{array}$ \\
\hline 1 & 0.6 & $2.5 / 2$ & Áluminum & 4.20 & 7 & 0.02 \\
2.8 & 0.8 & $2.5 / 1.5$ & Plexigl ass & 1.45 & 7 & 0.02 \\
\hline
\end{tabular}

Table 1. Model data

The models were so constructed that they can be mounted on the wind tunnel balance or brackets attached to the tunnel, or may be supported by the existing air bearing system for measurements under vibrating condition. At the central bulkhead of each model there are 32 pressure taps ( $d=0.025 \mathrm{in.}$ ) equally spaced around the circumference (Figure 2). Two taps were provided in the spanwise direction, at a distance of $4.5 \mathrm{in}$. and $9 \mathrm{in}$. from the mid-section, in the plane of the minor axis. The pressure taps are connected to plastic tubes of inside diameter $0.066 \mathrm{in}$. which are brought out from one end of the cylinder (Figure 1). In case of the plexiglass model it was thought advisable, due to the thin skin, to ascertain the rigidity of the 
panel. A simple test of deflection and natural frequency showed the panels to be of adequate stiffness with a maximum deflection of less than 0.0004 in. and natural frequency of $180 \mathrm{cps}$ during operating conditions.

The models were tested in a low speed, low turbulence, return type wind tunnel where the air speed can be varied from 4 - $150 \mathrm{ft} / \mathrm{sec}$ with a turbulence level less than $0.1 \%$. The pressure differential across the contraction section of $7: 1$ ratio can be measured on a Betz micromanometer with an accuracy of $0.2 \mathrm{~mm}$ of water. The test section velocity is calibrated against the above pressure differential. The rectangular cross-section, 36 in. $\times 27$ in., is provided with $45^{\circ}$. corner fillets which vary from 6 in. $x 6$ in. to 4.75 in. $\times 4.75$ in. to compensate partly for the boundary layer growth. The spatial variation of mean velocity in the test section is less than $0.25 \%$. The tunnel is powered by a 15 horsepower direct current motor driving a commercial axiflow fan with a Ward-Leonard system of speed control. An outline of the tunnel is shown in Figure 3. Figure 4 shows a model mounted in the wind tunnel during test. 

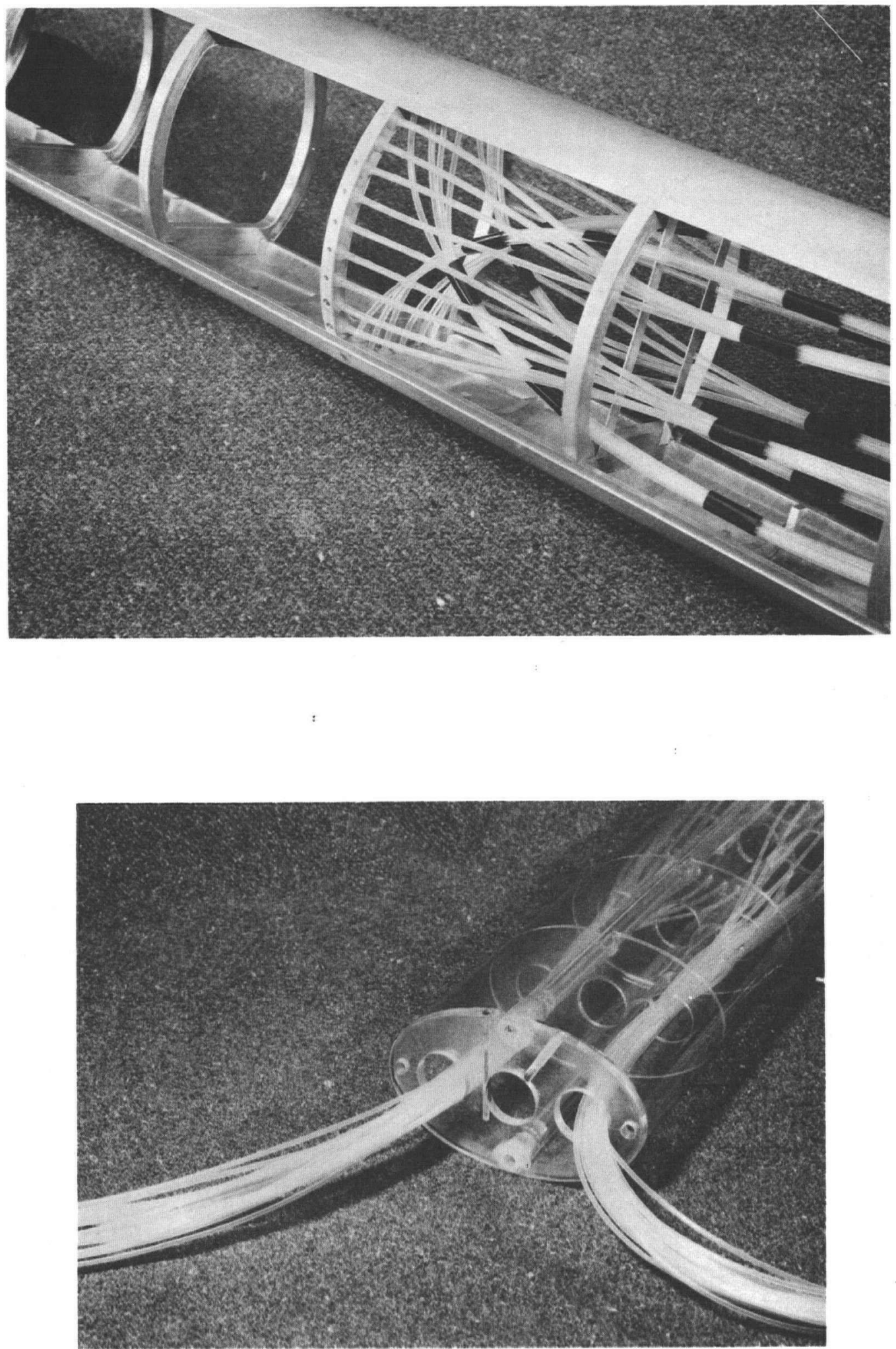

Figure 1. Constructional details of models 


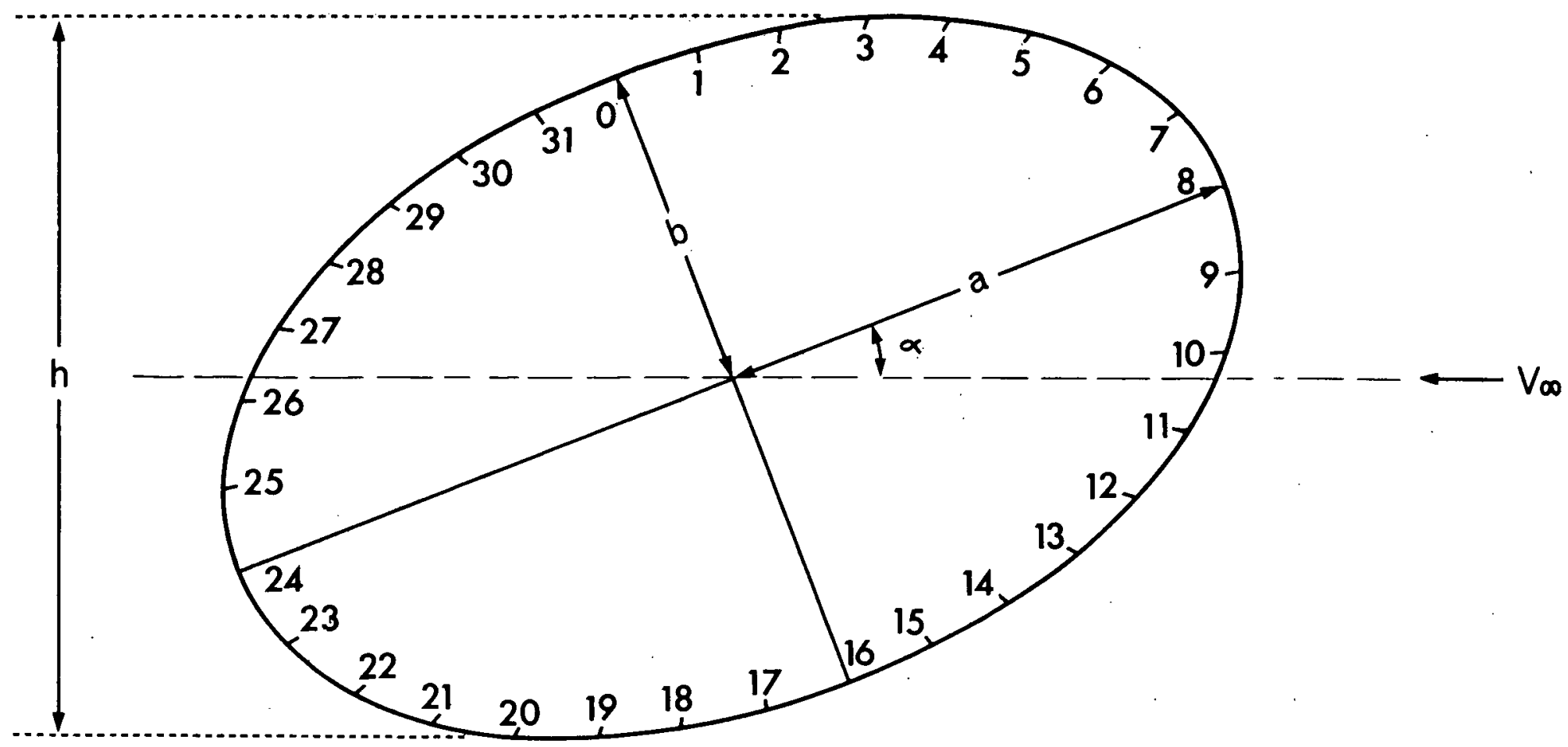

Figure 2. Numbering of pressure taps 


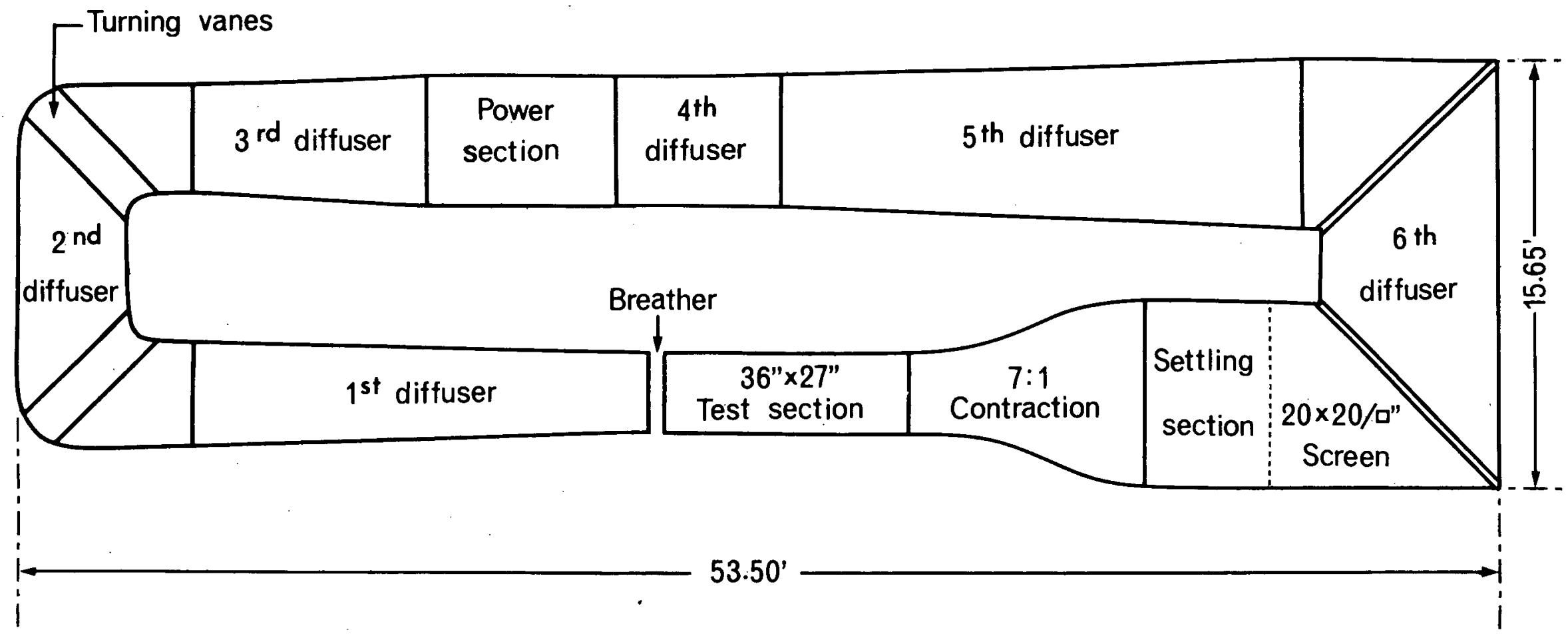

Figure 3." Wind tunnel outline 


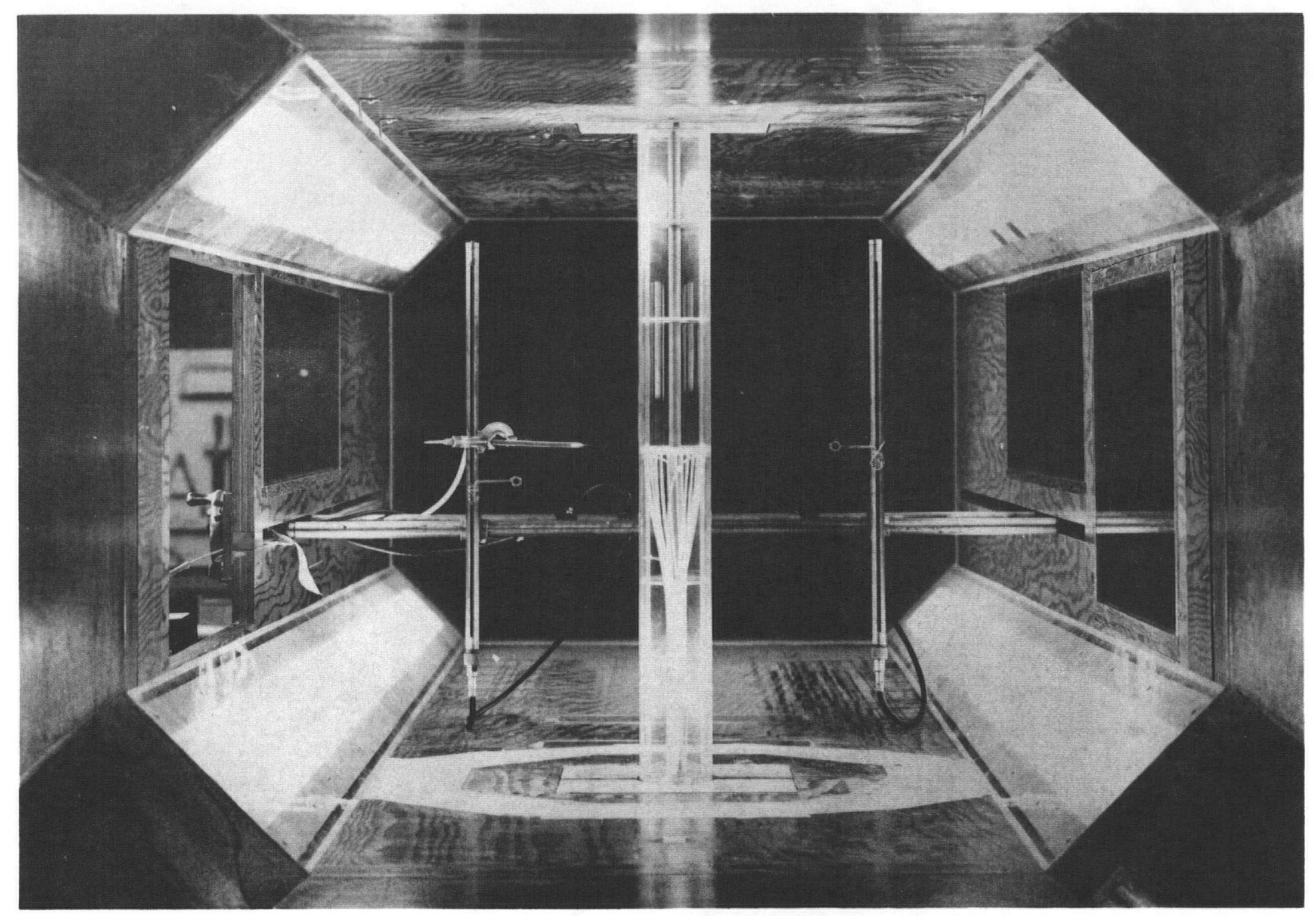

Figure 4. Wind tunnel test section with model 


\section{INSTRUMENTATION AND CALIBRATION}

\subsection{Pressure Transducer}

Recently a new pressure transducer, called Barocel Modular Pressure Transducing System, has appeared on the market. An extensive experimentation with the unit showed it to be quite suitable for the intended measurements. Consequentiy, two pressure transducers together with the necessary power and signal conditioner units were acquired. Developed by Datametrics Inc. of Waltham, Massachusetts, the Barocel is a high precision, stable capacitive voltage divider, the variable element of which is a thin prestressed stainless steel diaphragm (Figure 5). Positioned between fixed capacitor plates, the diaphragm deflects proportional to the magnitude of the applied pressure. An a.c. carrier voltage at $10 \mathrm{Kc}$. is applied to the stationary capacitor plates. The diaphragm attains a voltage level determined by its relative position between the fixed capacitor plates. With the Barocel appropriately arranged in a bridge circuit, the output voltage is determined by the ratio of capacitance of the diaphragm to each of the stationary electrodes. The carrier voltage is thereby amplitude modulated in accordance with the input pressure.

The units have 8 ranges. $0-10 \mathrm{~mm}$ of mercury on the least sensitive, and 0-0.001 mm of mercury on the most sensitive range. Further data given by the manufacturer are:

$\begin{array}{ll}\text { Output: } & 0-5 \text { volts d.c full scale } \\ \text { Linearity: } & \pm 0.1 \% \text { in ranges used } \\ \text { Accuracy of } & + \\ \text { Static Calibration: } & +0.5 \% \text { of full scale }\end{array}$




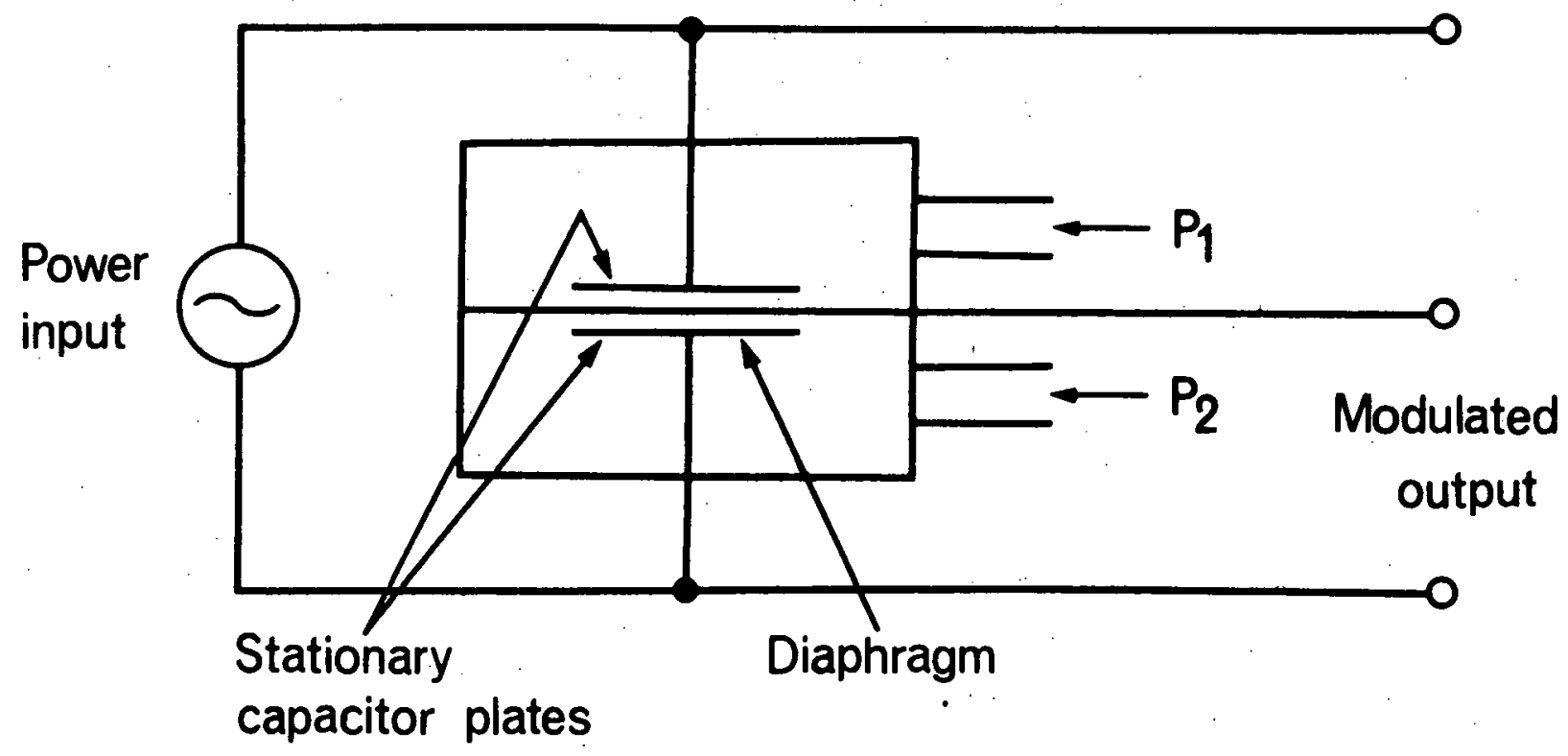

Figure 5. Schematic of Barocel pressure trans ducer 
Transient response: Typically less than 2 milliseconds to step input for line pressure of $750 \mathrm{~mm}$ of mercury

Stability: $\quad$ (a) $\pm 0.1 \%$ for $\stackrel{ \pm}{ \pm} 15^{\circ}$ ambient pressure change

(b) $\pm 0.01 \%$ for \pm 10 volts line voltage change

For (a) and (b) constant, $\pm 0.01 \%$ day to day

The Barocel is accurately calibrated for static pressures. For the fluctuating pressure signals transmitted through relatively long tubes considerable attenuation is to be expected. The attenuation of the signal will depend on such variables as tube length, tube diameter, number and size of constrictions (pressure taps), frequency and shape of the pressure signal and, in addition, the basic frequency response of the Barocel itself.

The inconsistencies in the results, obtained with the calibration methods previously developed in the department, were attributed to a resonance condition within the system and/or error in the theoretical prediction of pressure.

It was therefore realized that a system had to be developed where no resonance condition would exist between the transducer with tube and pressure tap on one side and the volume where the calibration signal was generated on the other side.

This condition was obtained with sufficient accuracy by using as a pressure source a 5 gallon drum with a thin latex diaphragm on one end. The pressure signal was generated by a baffle plate actuating the diaphragm. The baffle plate was driven by a vibration generator which in turn received its impulse from a function generator as shown in Figure 6. The natural frequency of the drum with diaphragm, baffle 
Function generator
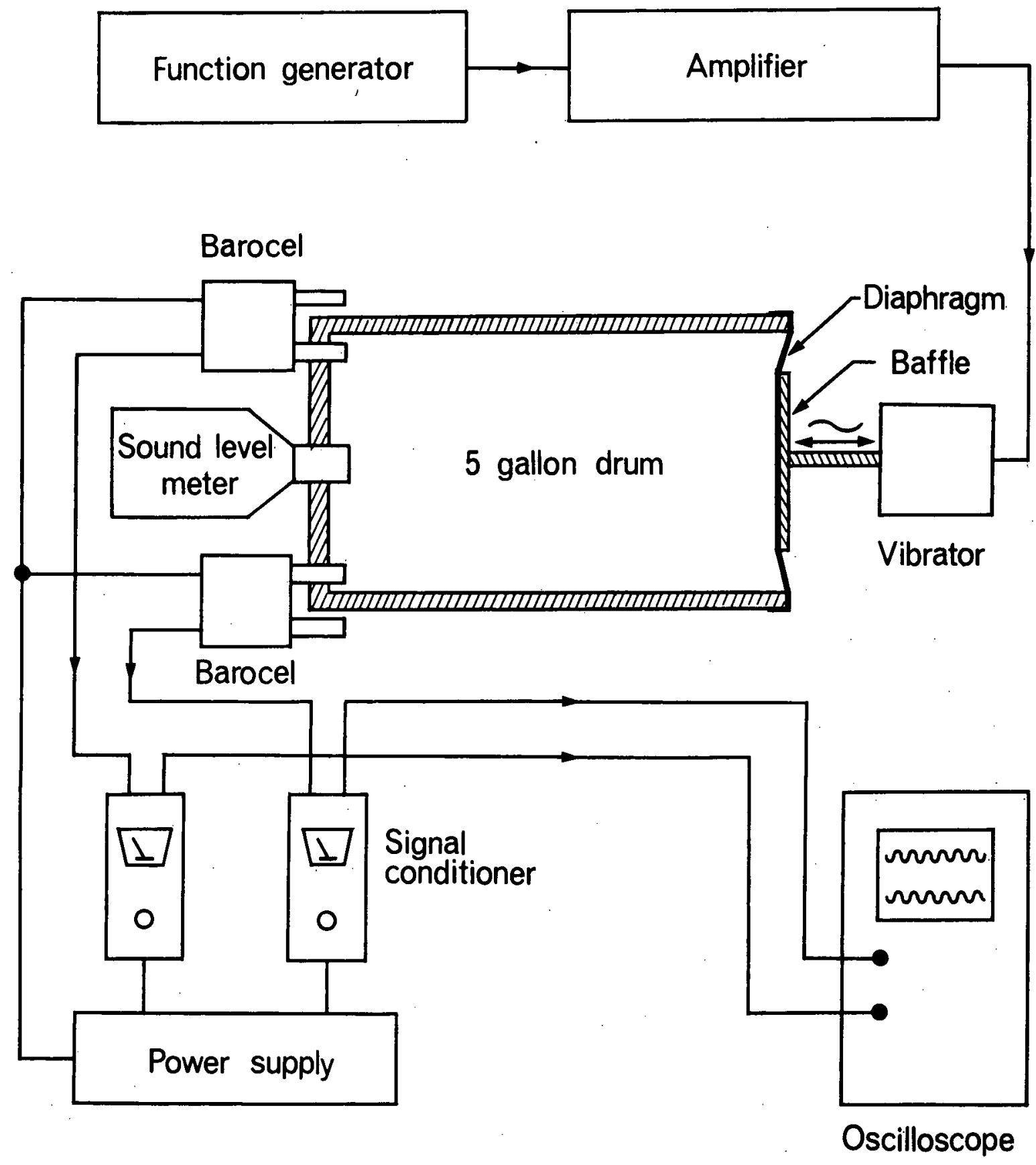

Figure 6. Block diagram of pressure generator and set-up for comparison of dynamical response 
and vibration generator was found to be $60 \mathrm{cps}$. However, this does not limit the use of the apparatus to lower frequencies provided the natural frequency of the transducer used to measure the source pressure is sufficiently high. To measure the source pressure a sound level meter could be used but these meters have a decibel scale, and an accuracy of $\pm 1 \mathrm{db}$ is equivalent to about $25 \%$ variation in the absolute pressure. Also, most sound leve1 meters have a poor frequency response below 20 cps.

This led to an investigation of the open port frequency response of the Barocels with a view to use one of them for measuring the calibration pressure at the source.

The natural frequency of the Barocel diaphragm itself is given by the manufacturer to be $2500 \mathrm{cps}$ or higher. Also, the transient response is specified to be less than $2 \mathrm{~ms}$. The Helmholtz resonator frequency of the cavity and the connection on one side of the diaphragm was calculated to be $290 \mathrm{cps}$. The experimental value obtained by actuating one side of the Barocel with a horn driver was found to be around $210 \mathrm{cps}$. Both these values are of the order of magnitude suggested by the transient response given above. It was established, through preliminary experiments, that the required frequency range would be from 5 to $35 \mathrm{cps}$. Considering the lower value of $210 \mathrm{cps}$ for the natural frequency, and a driving frequency of $40 \mathrm{cps}$, one would get an output within $\pm 4 \%$ of the input if a linear system with damping coefficient somewhere between zero and critical is assumed. This justifies the use of the statically calibrated Barocel for measuring the pressure inside the drum. Figure 6 illustrates the 
set-up of the pressure generator during the initial evaluation of the Barocel response. It was found that for sinusoidal inputs the signals from the Barocel and the function generator could be superimposed on the oscilloscope. Even in the extreme cases of square and triangular wave inputs the response of the calibration system was quite good as indicated in Figure 7 . The results of the initial calibration with varying tube length and frequency are shown in Figure 8. The approximate exponential decay of output amplitude with tube length is as expected $\mathrm{d}^{20}$. The appearance of "bumps" at shorter tube lengths, most pronounced at $15 \mathrm{cps}$, cannot be attributed to a resonance condition between the drum and the Barocels. The same phenomena will occur under actual measuring condition in the wind tunnel, and it may be considered as a peculiarity of this combination of tube, pressure tap and transducer.

Since noticeable attenuation occurred due to the presence of a constriction in the tube all calibrations were performed with a pressure tap in the circuit. The calibration plots for a Barocel when connected to a pressure tap through a tube of given length and diameter are shown in Figure 9. This also demonstrates the linearity of the system.

For work in the wind tunnel it was found impossible to use atmospheric pressure as reference. This is because the difference between the static pressure at the tap and the atmospheric pressure was found to be so large as to throw the Barocel off scale at sensitive settings. Moreover, surges in the base pressure affected the pressure field around the model and gave rise to the same effect. Hence, the 


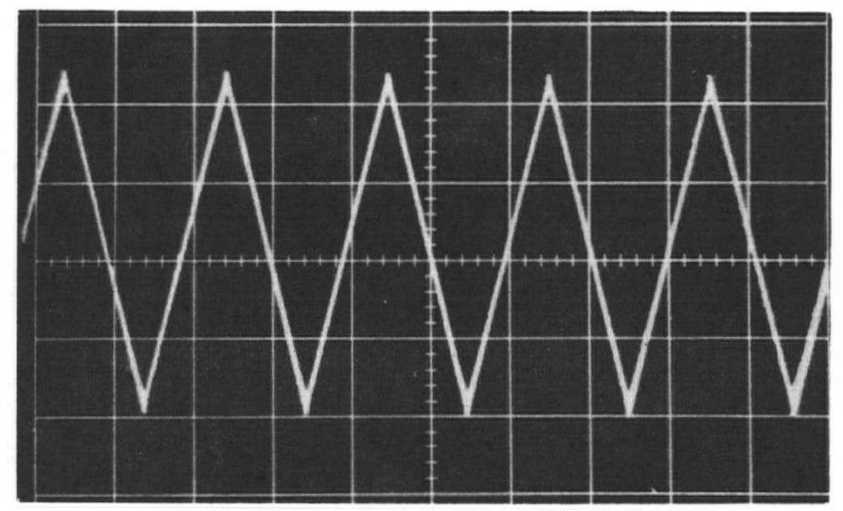

(a)

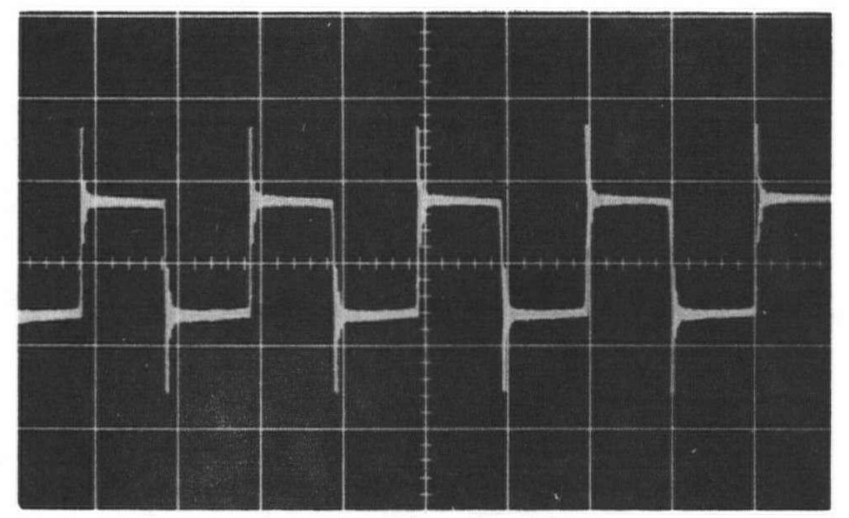

(b)

Figure 7. Response of calibration system to different input wave forms ( $1 \mathrm{cps}, 0.0016 \mathrm{psi}$ ).

(a) Triangular wave input

(b) Square wave input 


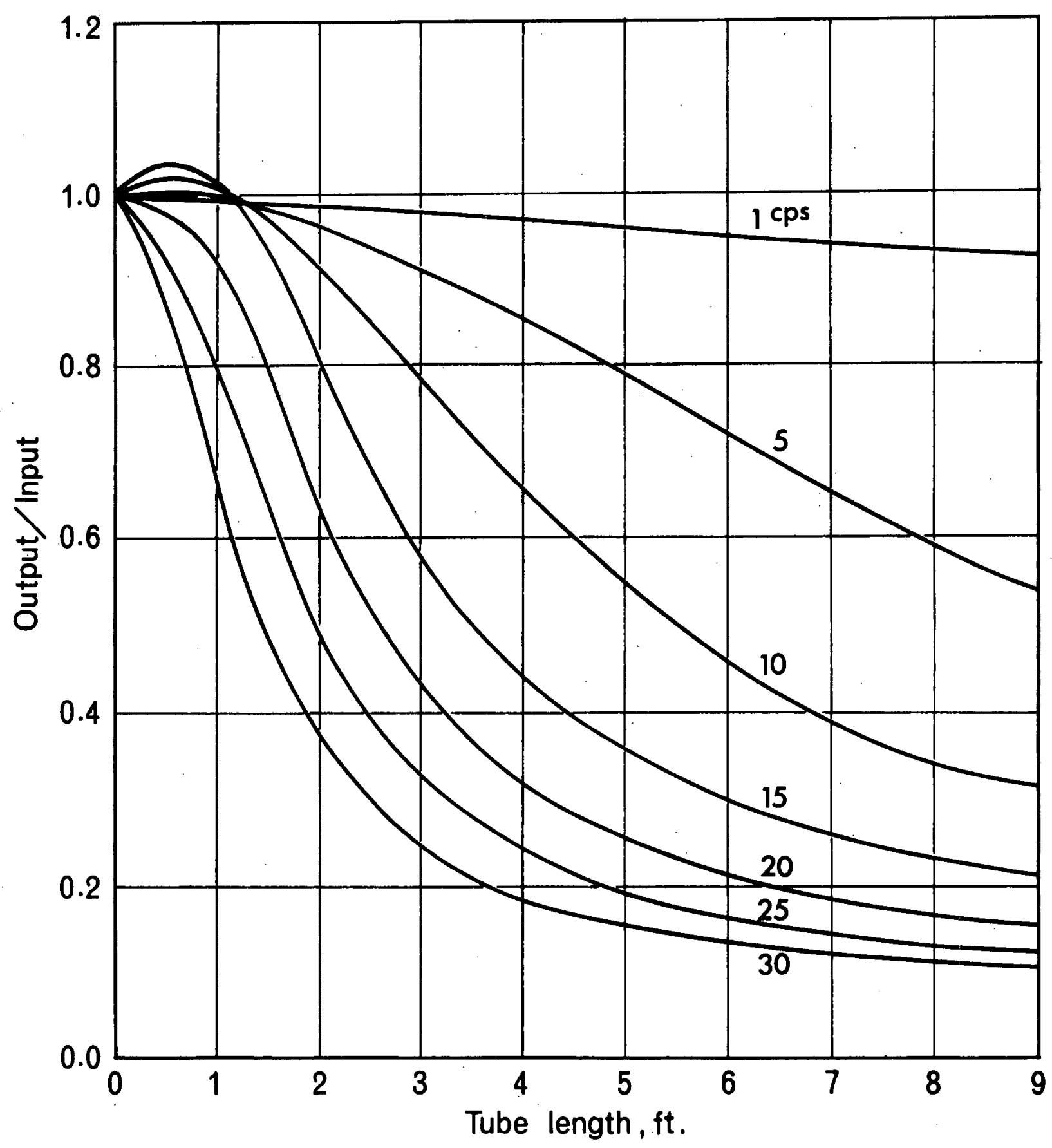

Figure 8. Pressure attenuation as a function of tube length and frequency (tube diameter $=$ 0.066 in.) 


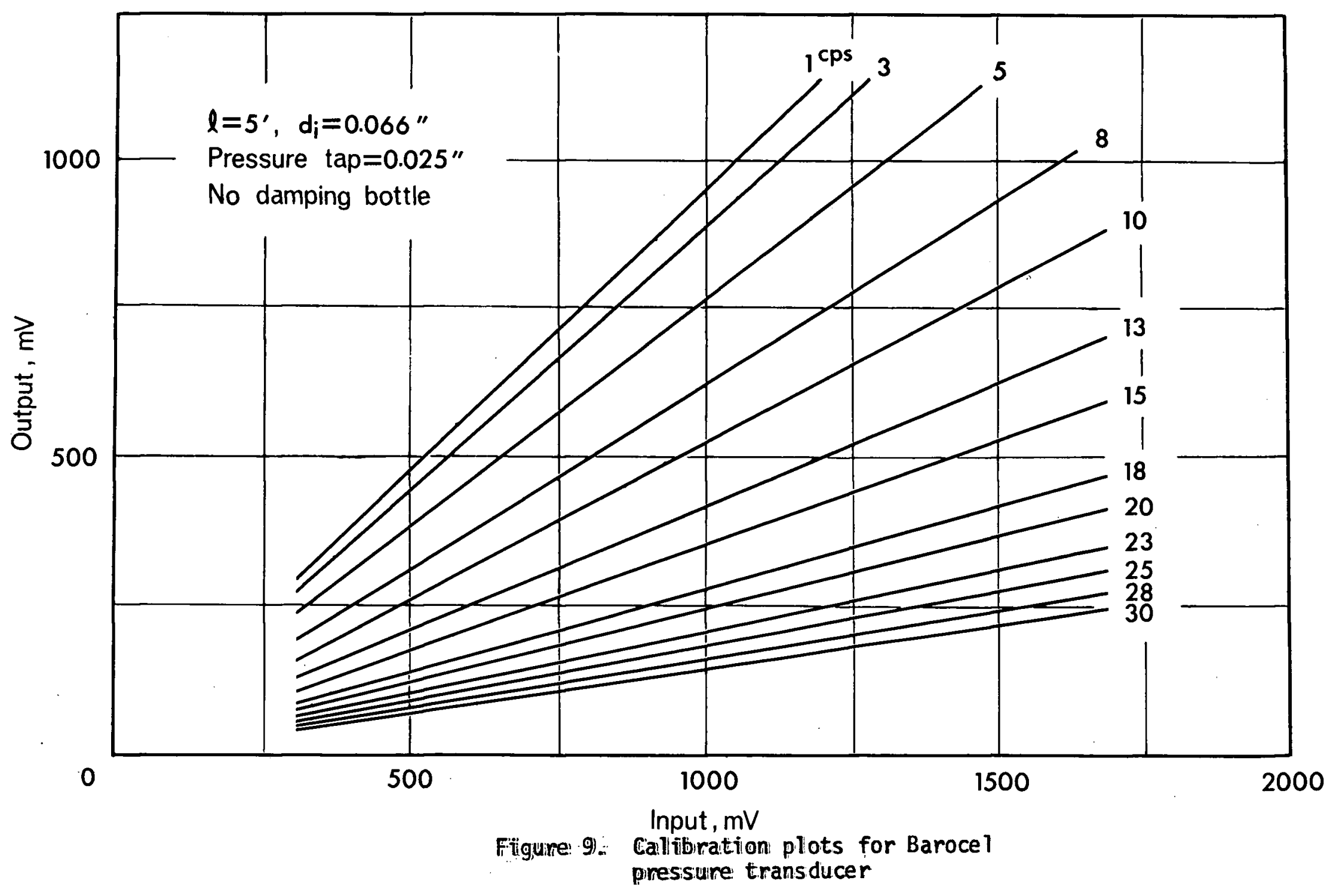


final calibration set-up incorporated a damping volume between the pressure ports of the Barocel, thus using the static pressure of the tap in question as reference. A block diagram and a photograph of the calibration set-up are shown in Figure 10 and 11 respectively. The effect of amplitude and frequency on output, with the damping bottle in the circuit, is shown in Figure 12. For convenience the calibration curves in Figures 9 and 12 are plotted in Figure 13 as a ratio of output to input. It can be noticed that above $15 \mathrm{cps}$ there is no attenuation due to the damping bottle. The specific tube length and diameter were chosen for practical reasons. Sinusoidal signals were used throughout the calibration.

\subsection{Manometer}

An inclined Lambrecht manometer with ethyl aicohol was used to measure static pressure on the surface of the model. The manometer can be read with an accuracy of 1/10th of a millimeter. It was found necessary to reduce the fluctuations of the liquid column caused by the pressure surges previously mentioned. The constrictions in the tubing formed by four hypodermic needles (\#19) in series gave adequate damping.

\section{3 Wake Probe}

The wake survey was carried out using a disc probe constructed by Ferguson ${ }^{16}$ and described in detail by Bryer et $a 1^{21}$. As pointed out by these investigators, the probe is relatively insensitive to 


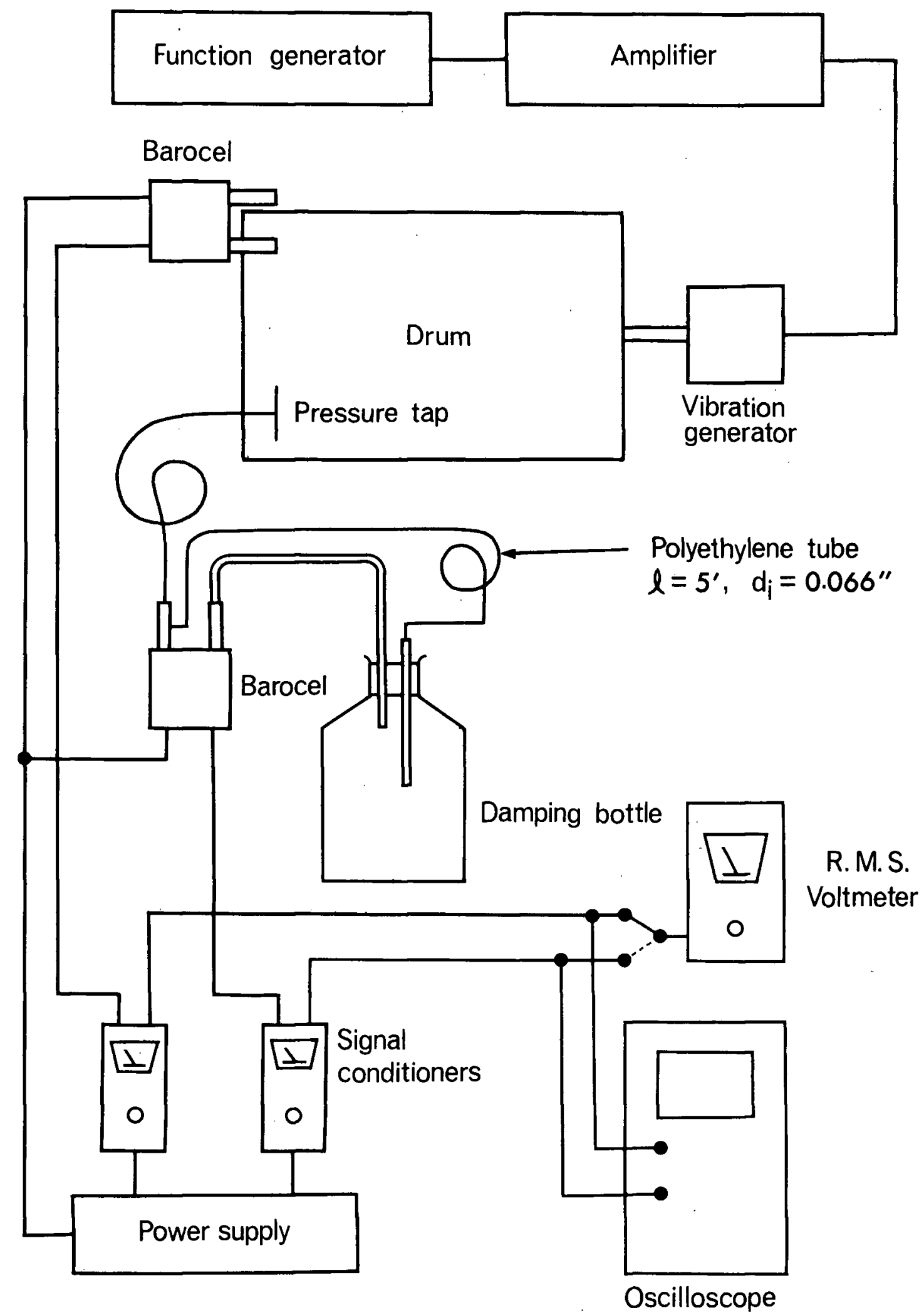

Figure 10. Block diagram of the calibration apparatus 


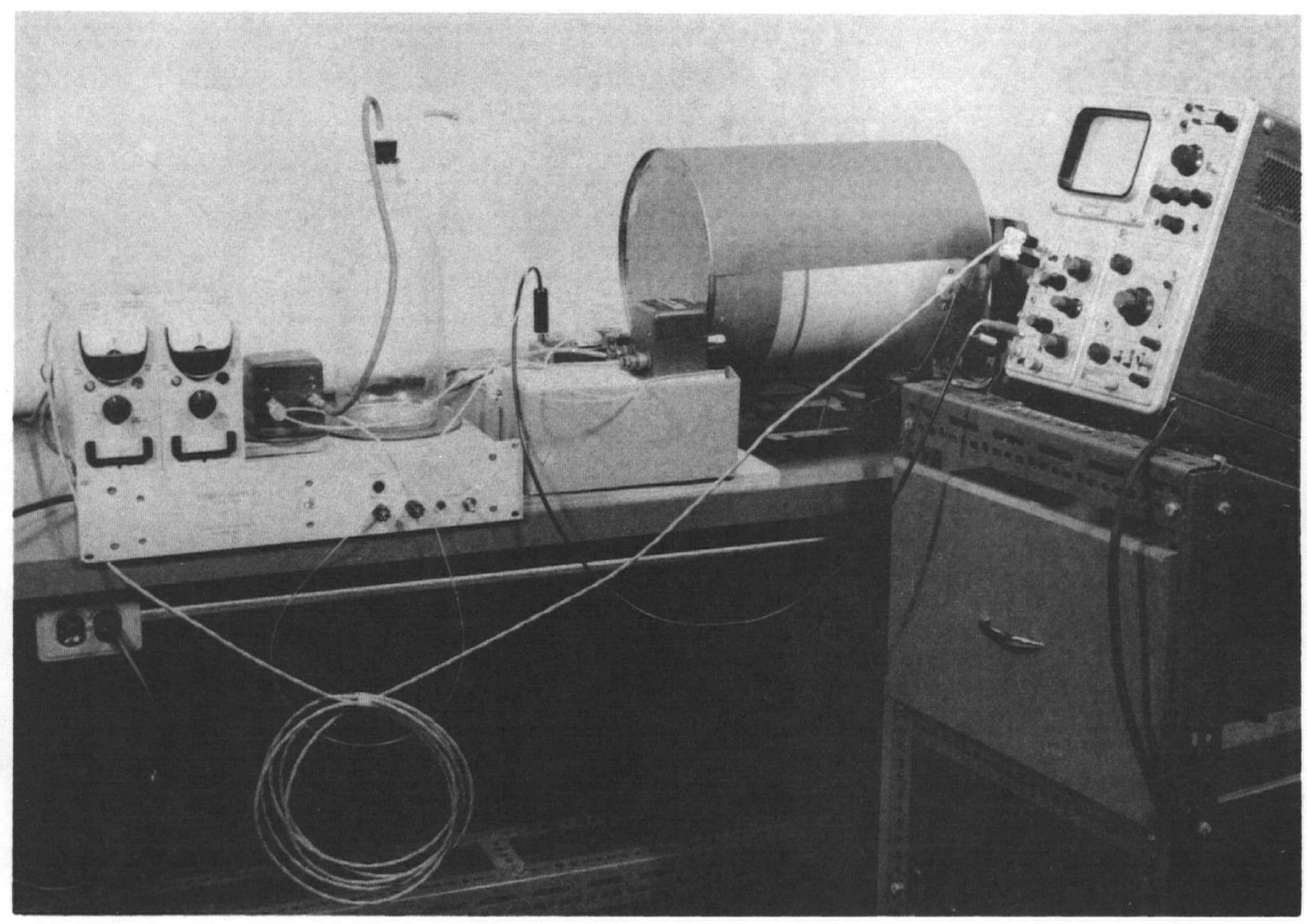

Figure 11. Calibration apparatus 
o

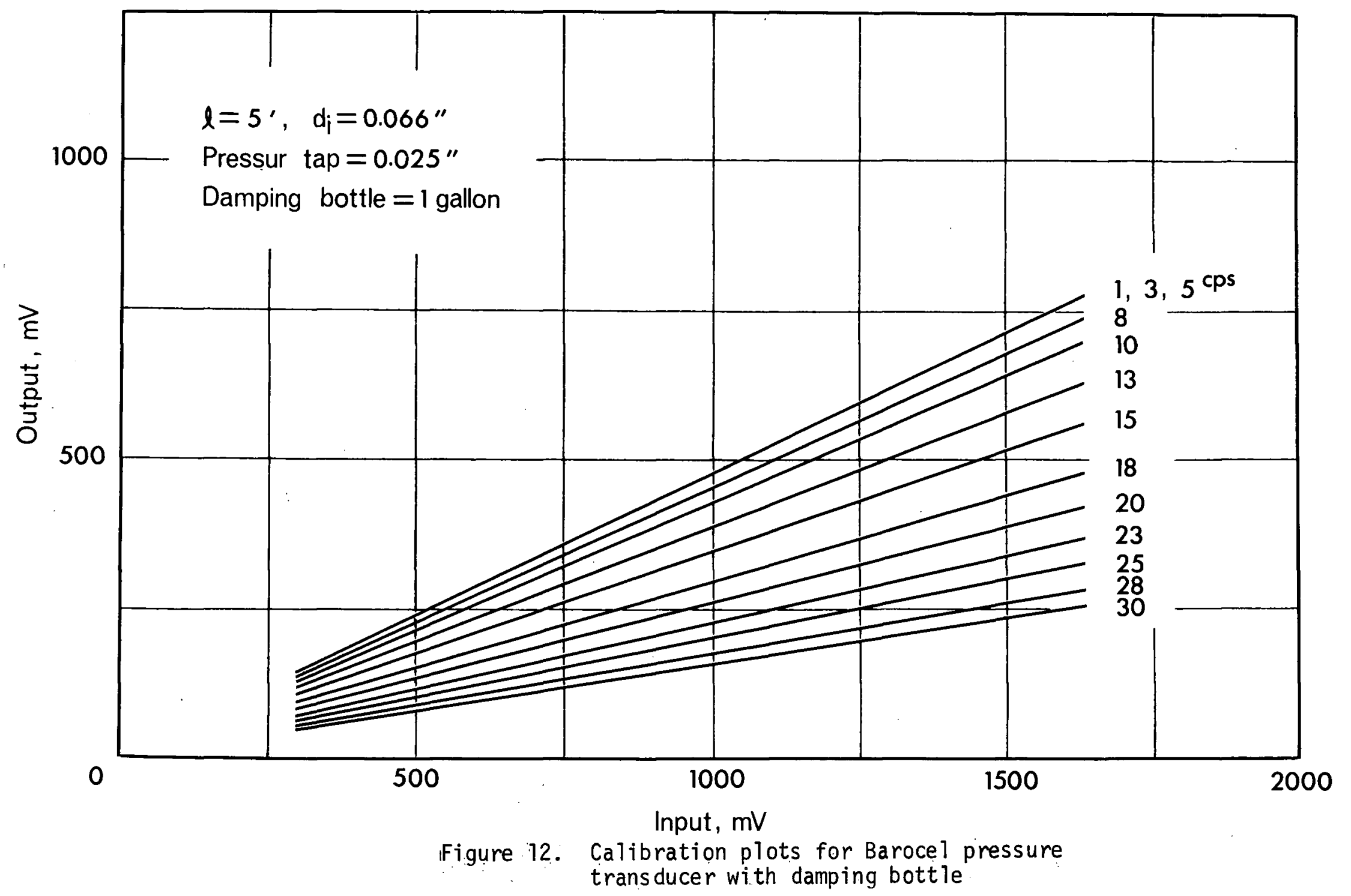




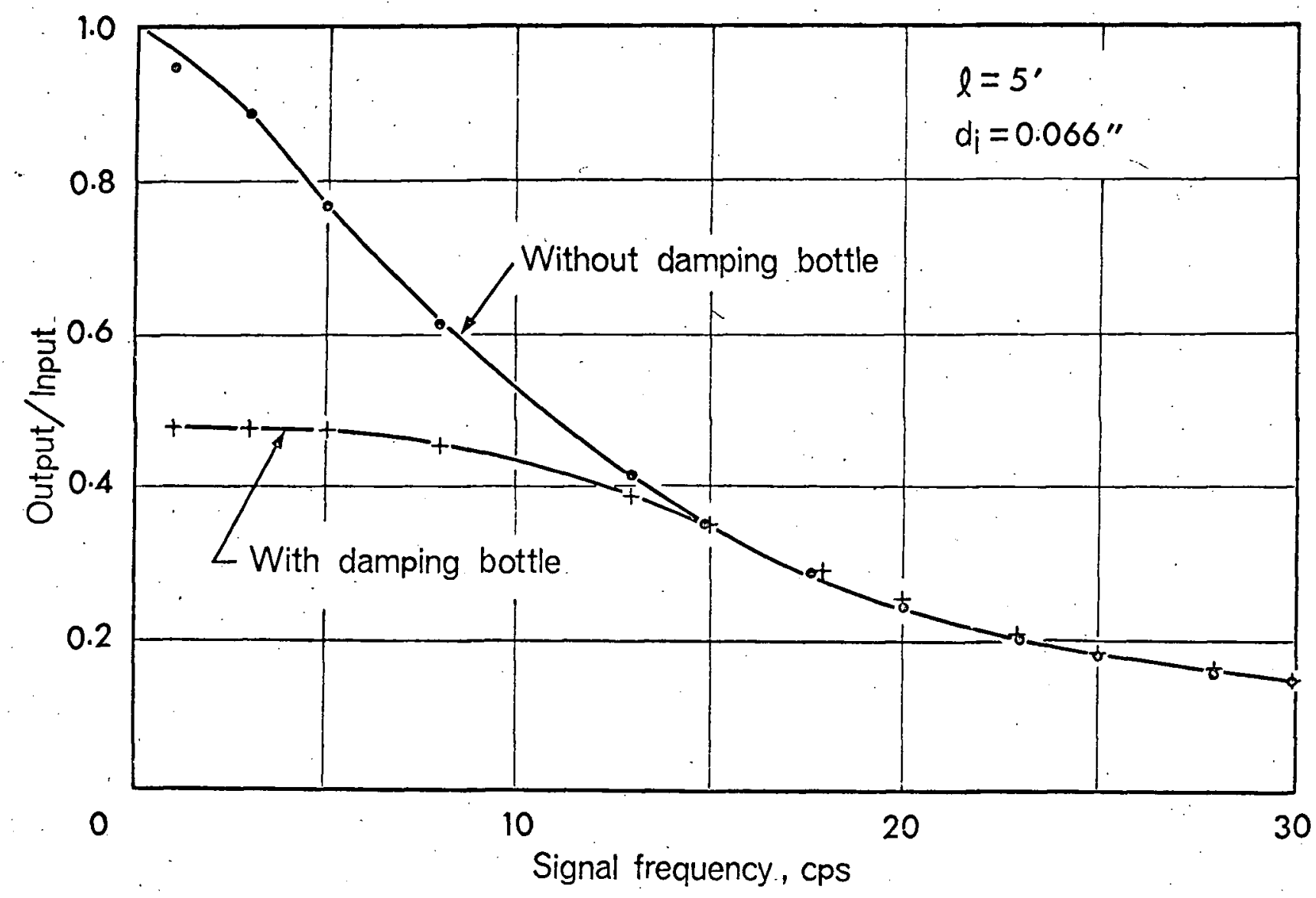

Figure 13. Comparison between calibration curves for Barocel pressure transducer with and without damping bottle 
pitch $\left( \pm 4^{\circ}\right)$ and yaw $\left( \pm 20^{\circ}\right)$. The main dimensions of the probe are given in Figure 14.

\subsection{Band Pass Filter}

There are several sources of disturbances, e.g. fan drive, other laboratory equipment, surges in the tunnel, etc., which superpose undesirable pressure variations on that created by the shedding vortices. In general, the intensity of the 'noise' may be considered constant. On the other hand the pressure fluctuations due to shedding vortices depend on location of the tap and attitude of the model. This being the case there were situations where the noise had a tendency to overshadow the vortex-generated pressure variations. It was, therefore, necessary to introduce a band pass filter in the pressure measuring system to eliminate the undesirable noise. The typical pressure traces of filtered and unfiltered signals are compared in Figure 15. At $\alpha=0$, where the signals are weak in relation to noise, the necessity of using a filter is obvious. At $\alpha=90^{\circ}$, where the signals are more powerful, the noise level becomes relatively insignificant and the filter is no longer essential. It was also observed that the pressure signal from the separated flow region was slightly more: irregular than that from the laminar field. This may be due to nigher turbulence level in the wake.

During measurements the filter was calibrated for every change affecting the vortex shedding frequency. Operating the filter at mid-band frequency and with the high and low cut-off settings separated by a factor of 1.5 gave an attenuation between 0.8 and 0.9 . This was 
Hypodermic needle

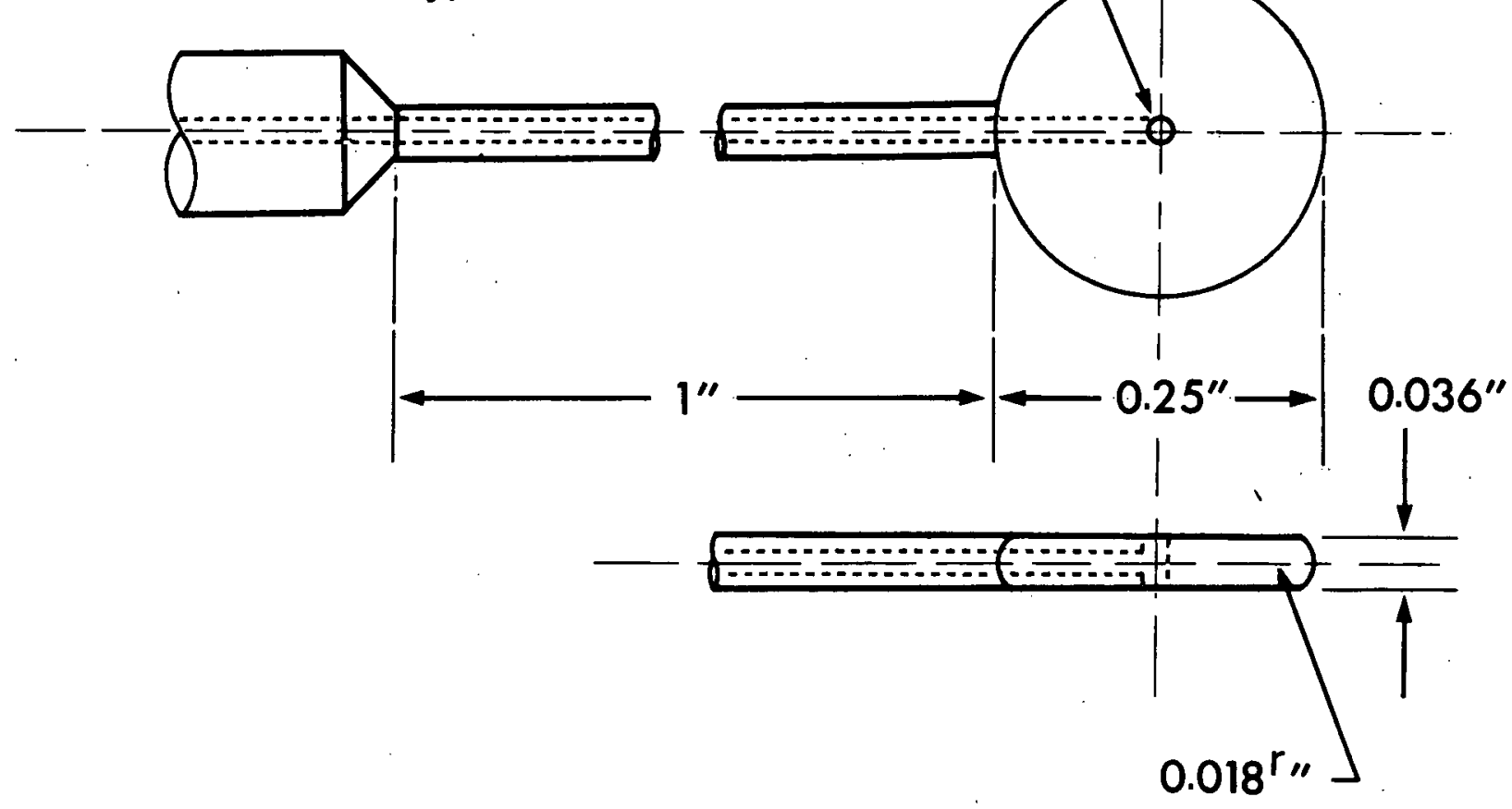

Figure 14. Geometry of the disc probe 


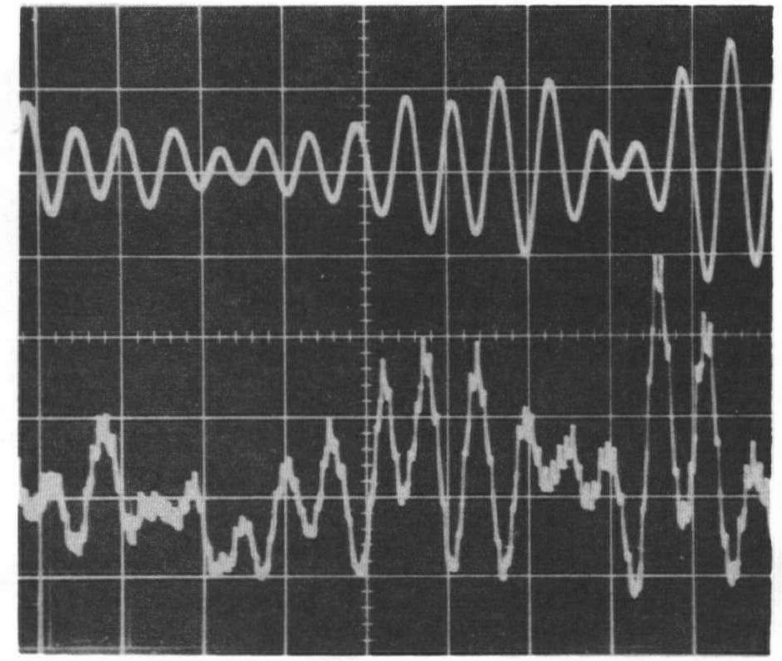

$e=0.6$

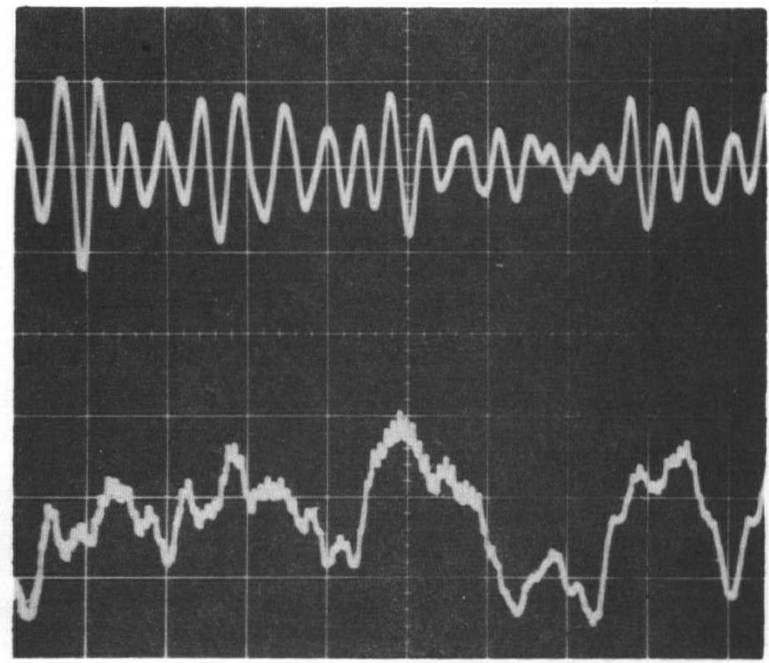

$e=0.8$

(a)

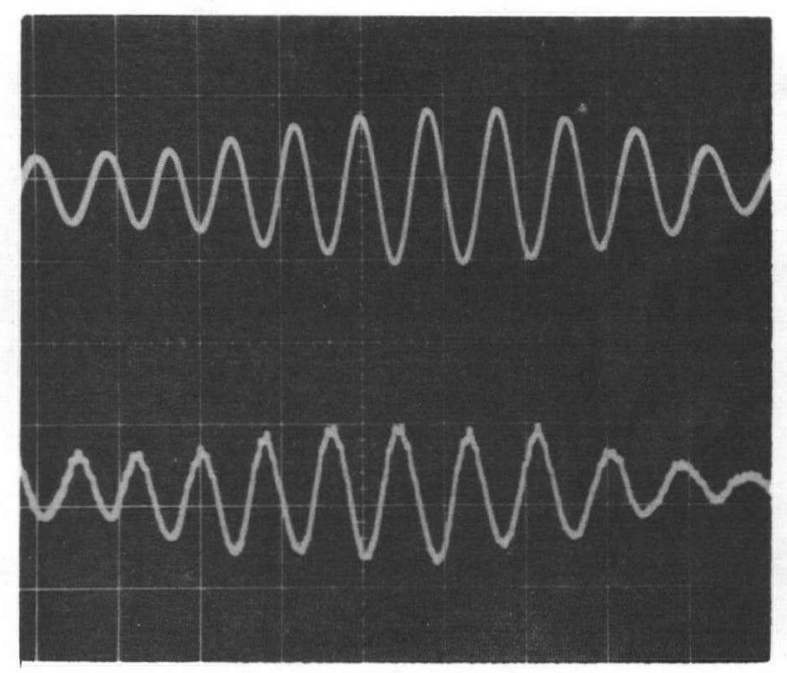

$\mathrm{e}=0.6$

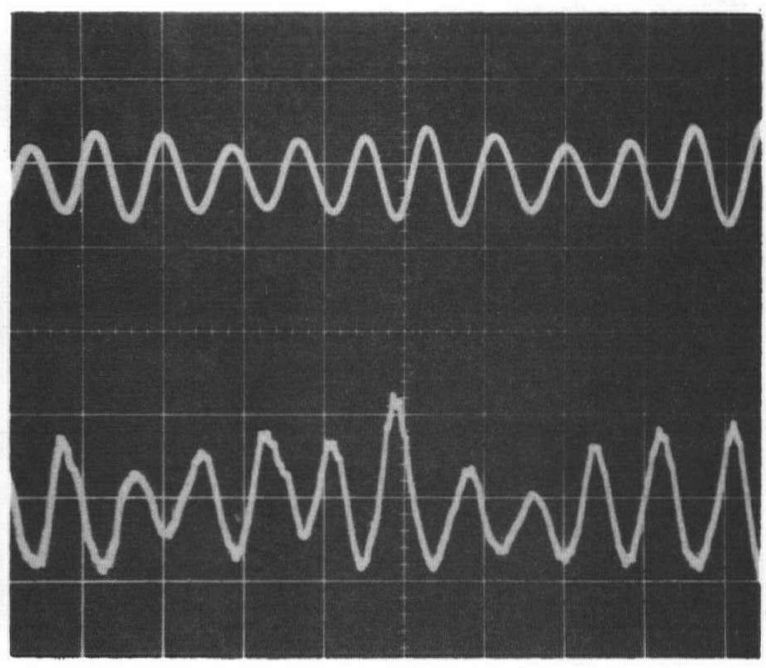

$\mathrm{e}=0.8$

(b)

Figure 15. Typical fluctuating pressure traces (not to the same scale)
(a) $\alpha=0$,
(b) $\alpha=90^{\circ}$

Upper trace represents filtered signal 
found by feeding a sinusoidal signal from the function generator to the rms voltmeter and measuring the difference in output with and without the filter.

\subsection{R.M.S. Voltmeter}

Due to considerable, seemingly random, amplitude modulations of the fluctuating pressure it was necessary to present the results as time-average values. Under certain conditions several minutes of averaging was required to get reproducible results. This led to the necessity of using a true rms voltmeter converting the fluctuating pressure signal to an equivalent d.c. signal. An external $r$-c damping circuit was used to reduce the variations caused by the amplitude modulations. The final steady d.c. level was then measured on the vacuum tube voltmeter. Because of the extremely high input resistance of the latter no measurable voltage drop occurred over the damping resistors (Figure 16).

\subsection{Electronic Instruments}

Following is the list of the electronic apparatus used in the experimental work:

Filters: Krohn-Hite, band pass variable filter, models $330 \mathrm{~B} \& 330 \mathrm{~A}$.

Voltmeters: Hewlett Packard, HP-3400A true rms voltmeter, and $\mathrm{HP}-412$ vacuum tube voltmeter.

Function Generator: Hewlett Packard, low frequency function generator, model 202A. 
Vibration Generator: Goodmans, type V47.

Oscilloscope: Tectronix, type 564, dual trace storage oscillos cope.

Chart Recorder: Honeywe11, 906c visicorder.

Low frequency ampli-

fier with power

supplies:

Built in the department ${ }^{22}$.

R-C damping circuit: Built in the department (Figure 16). 
HP - 3400A

R.M.S. Voltmeter

Output resistance $1 \mathrm{~K} \Omega$

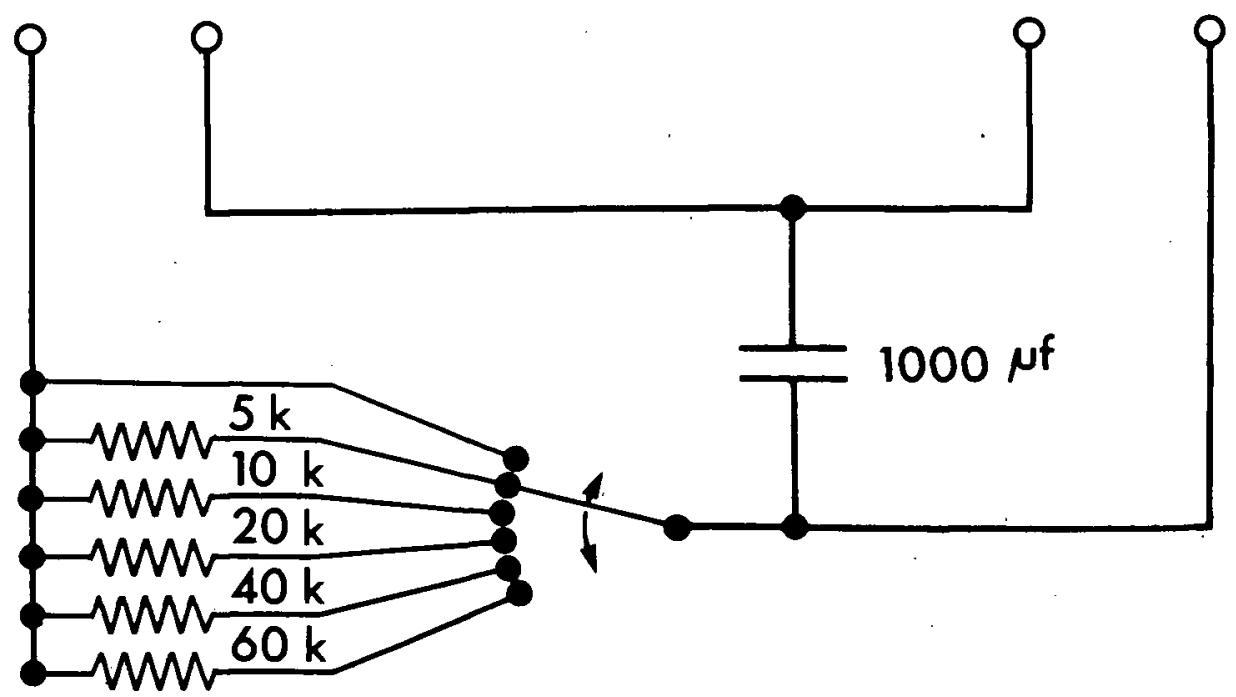

$H P-412 A$

V.T. Voltmeter

Input resistanc $200 \mathrm{M} \Omega$

Figure 16. $R-C$ damping cireuit 


\section{TEST PROCEDURES}

\subsection{Fluctuating Pressure Measurements}

The diagrammatic lay-out of the apparatus used for fluctuating pressure measurements is given in Figure 17 . During measurements the $r-c$ damping circuit was set to give minimum variation of the voltmeter reading. The fluctuating pressure signal was also displayed on the oscilloscope to determine the maximum amplitude and Strouhal frequency.

\subsection{Phase Measurements}

The phase between the fluctuating pressures at different taps was obtained by feeding the signals to the Visicorder and measuring the average phase shift over 10 cycles. The effect of any phase shift in the instrumentation was nullified by measuring all phase shifts from a permanent reference tap at $90^{\circ}$ to the wind direction. The data showed considerable scatter which increased with the distance from the reference tap. Figure 18 shows, schematically, the arrangement of the instrumentation for phase measurements. The set-up during a typical test run is shown in Figure 19.

\subsection{Mean Static Pressure Distribution}

Mean pressure on the model was measured using a Lambrecht manometer: One leg of the manometer was connected to a total head tube in the settling chamber and the other to the pressure tap on the mode 1 . 


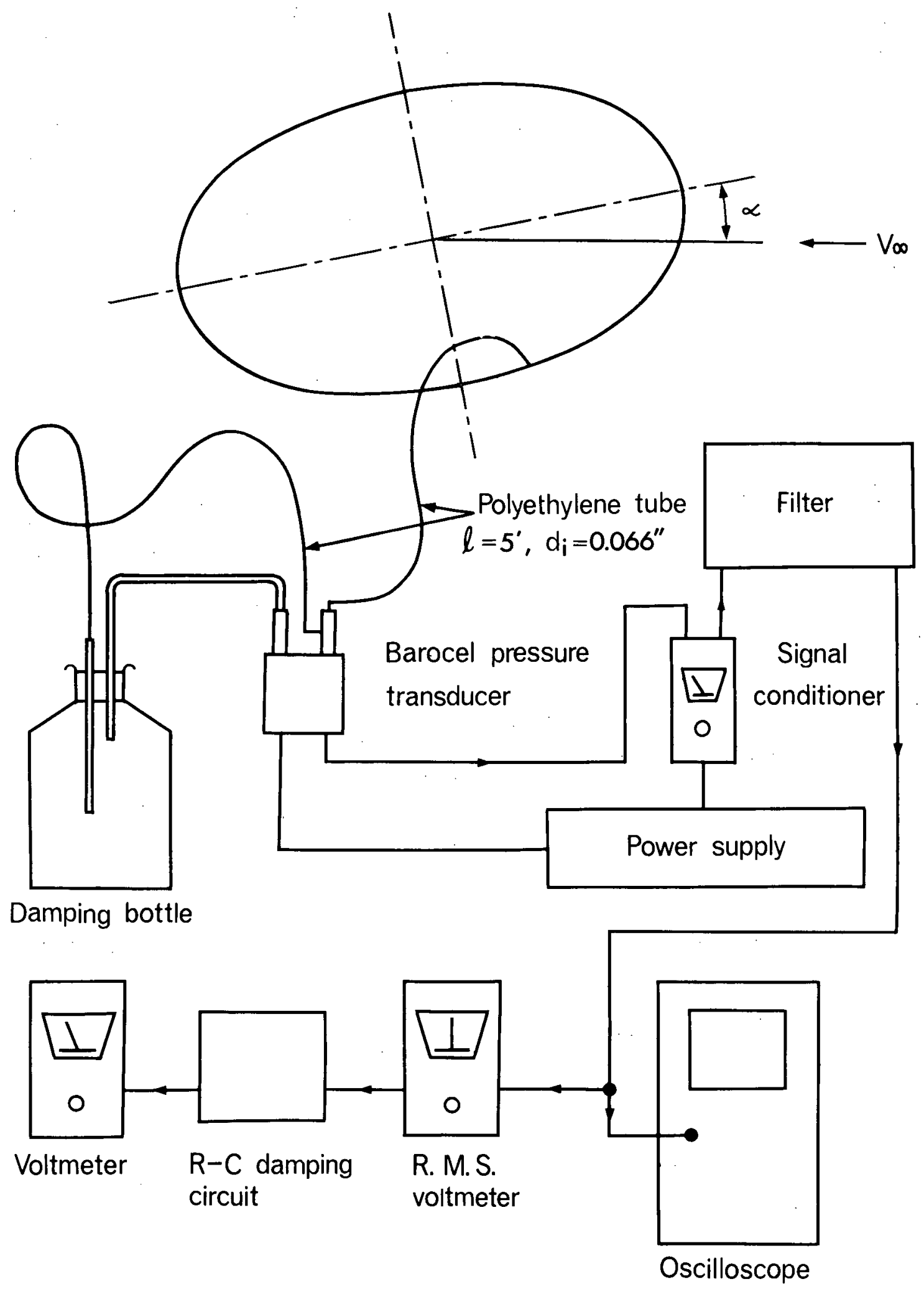

Figure 17. Block diagram of the fluctuating pressure measuring set-up 


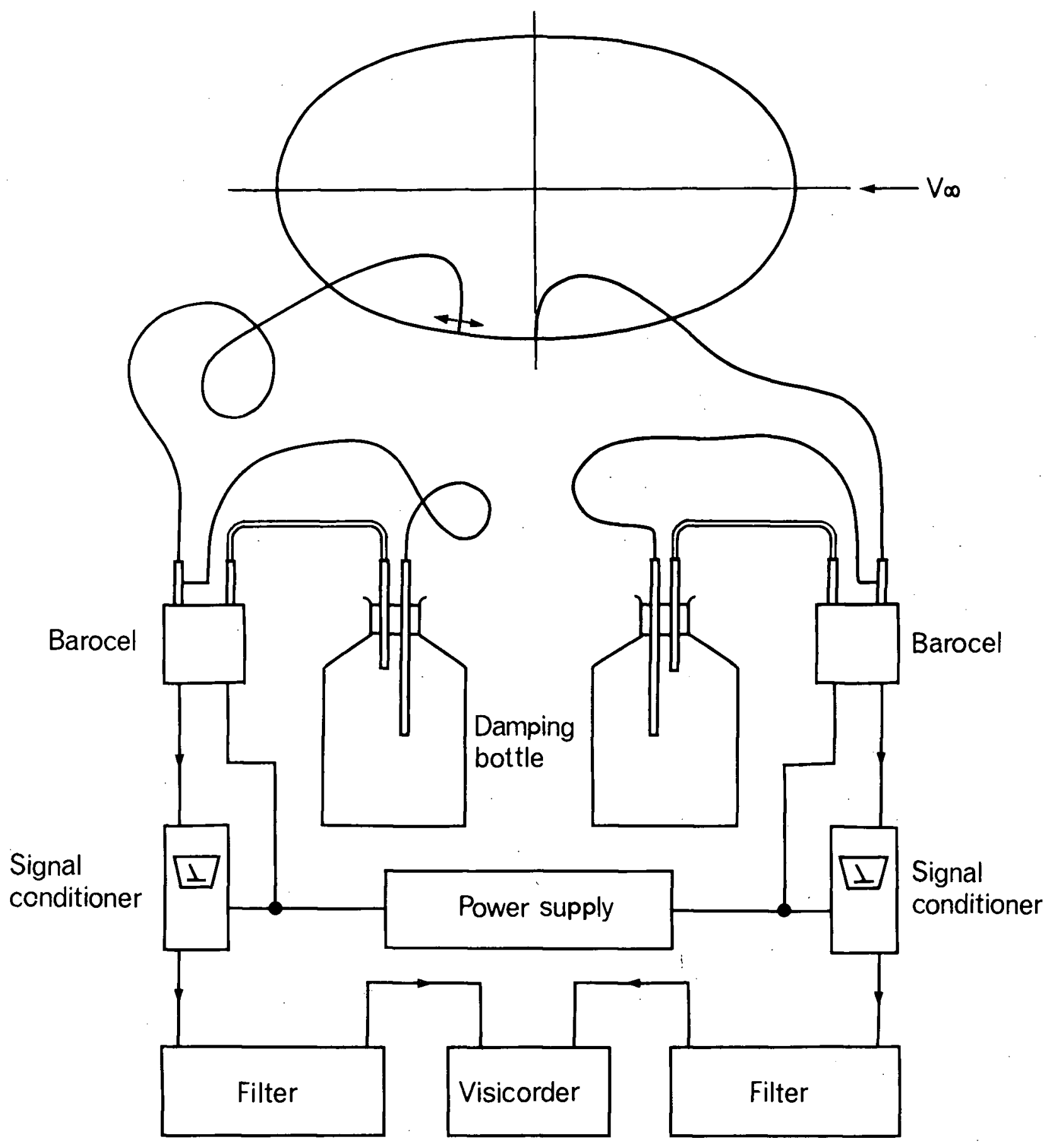

Figure 18. Block diagram of the phase measuring system 


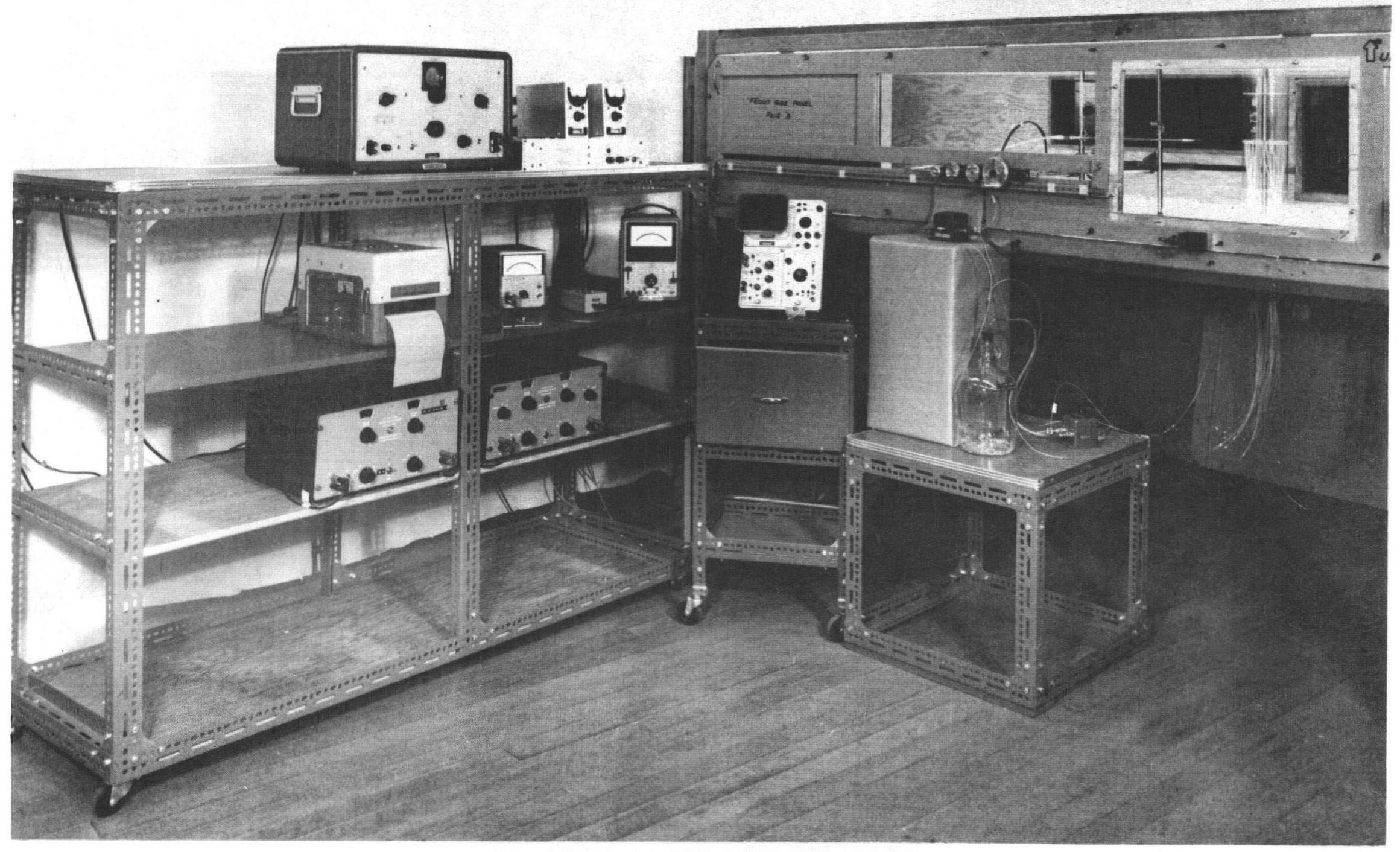

Figure 19. Instrumentation set-up during a typical test run 


\subsection{Wake Measurements}

The determination of wake geometry was accomplished using the method described by Heine ${ }^{15}$ and Fergus on ${ }^{16}$. The transverse distance between the vortex centrelines was measured by the wake probe connected to the pressure transducer in a manner similar to that shown in Figure 17. A photograph of the wake probe and the pressure transducers connected to the damping bottles is given in Figure 20: Moving the probe across the wake and plotting the rms value of the signal gave two peaks at the vortex centrelines.: The distance between these peaks represents the lateral spacing between the vortex core lines at that station. The longitudinal spacing between the consecutive vortices was obtained by using a pressure tap as reference and moving the probe downstream on the same side of the model until the signals were $180^{\circ}$ out of phase. From this position the probe was moved further downstream so that the the signals were in phase. The process was repeated until limited by the travel of the traversing gear. Twice the distance between two successive measurements gave the desired longitudinal spacing between the vortices. The instrument arrangement was similar to the one used for phase measurements (Figure 18), except that the signals were displayed on the oscilloscope. 


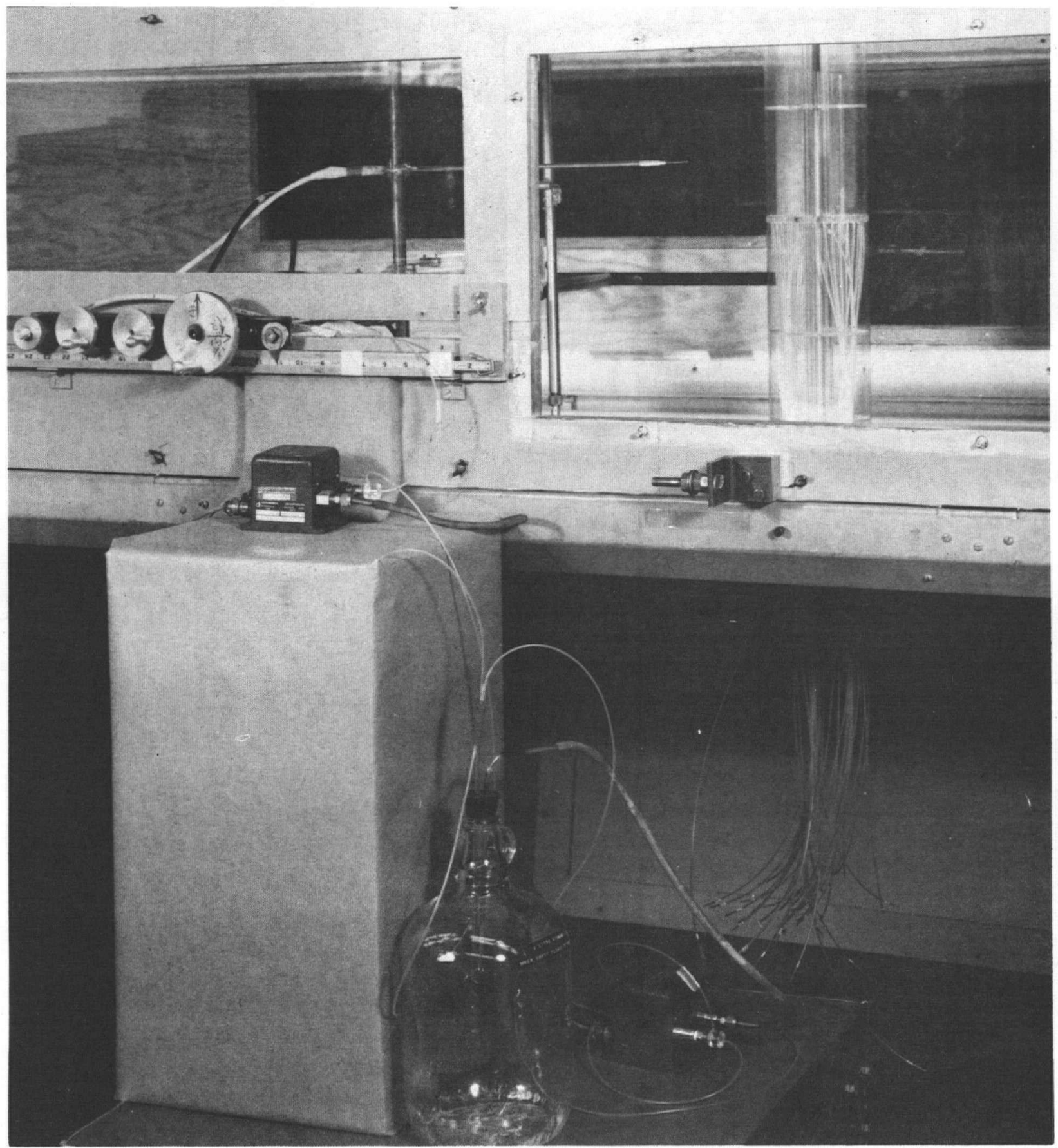

Figure 20. Traversing gear with probe during wake measurements 


\section{TEST RESULTS AND DISCUSSION}

\subsection{Strouhal Number}

The Strouhal frequency was measured in the Reynolds number range of $2 \times 10^{4}-10^{5}$ for three different angles of attack, and it was observed to be linear with increasing wind speed as shown in Figure 21. The effect of angle of attack on the Strouhal number was also obtained in the same Reynolds number range. These variations based on the minor axis as well as the projected height are shown in Figure 22.

The Strouhal number based on projected height $(h)$ showed comparatively less dependence on the angle of attack $(\alpha)$ in the Reynolds number range investigated. An observation concerning the Strouhal number variation at low angles of attack is pertinent here. For the thicker ellipse a slow rise in the projected height with a small drop in shedding frequency leads to an almost uniform Strouhal number in this range. On the other hand, for the thinner ellipse the very sharp drop in shedding frequency off-sets the relatively greater rise in projected height thus leading to a decrease in Strouhal number. The similar tendency was also observed by Schramm ${ }^{17}$ who carried out Strouhal number measurements for the ellipses of four different eccentricities $(e=0.662,0.866,0.969,0.998)$. In a11 cases except one the measurements were limted to $0-45^{\circ}$, range due to, as reported by him, "lack of stable vortex street". This is in contrast to the strong and well defined fluctuating signals observed in the test results presented here. 


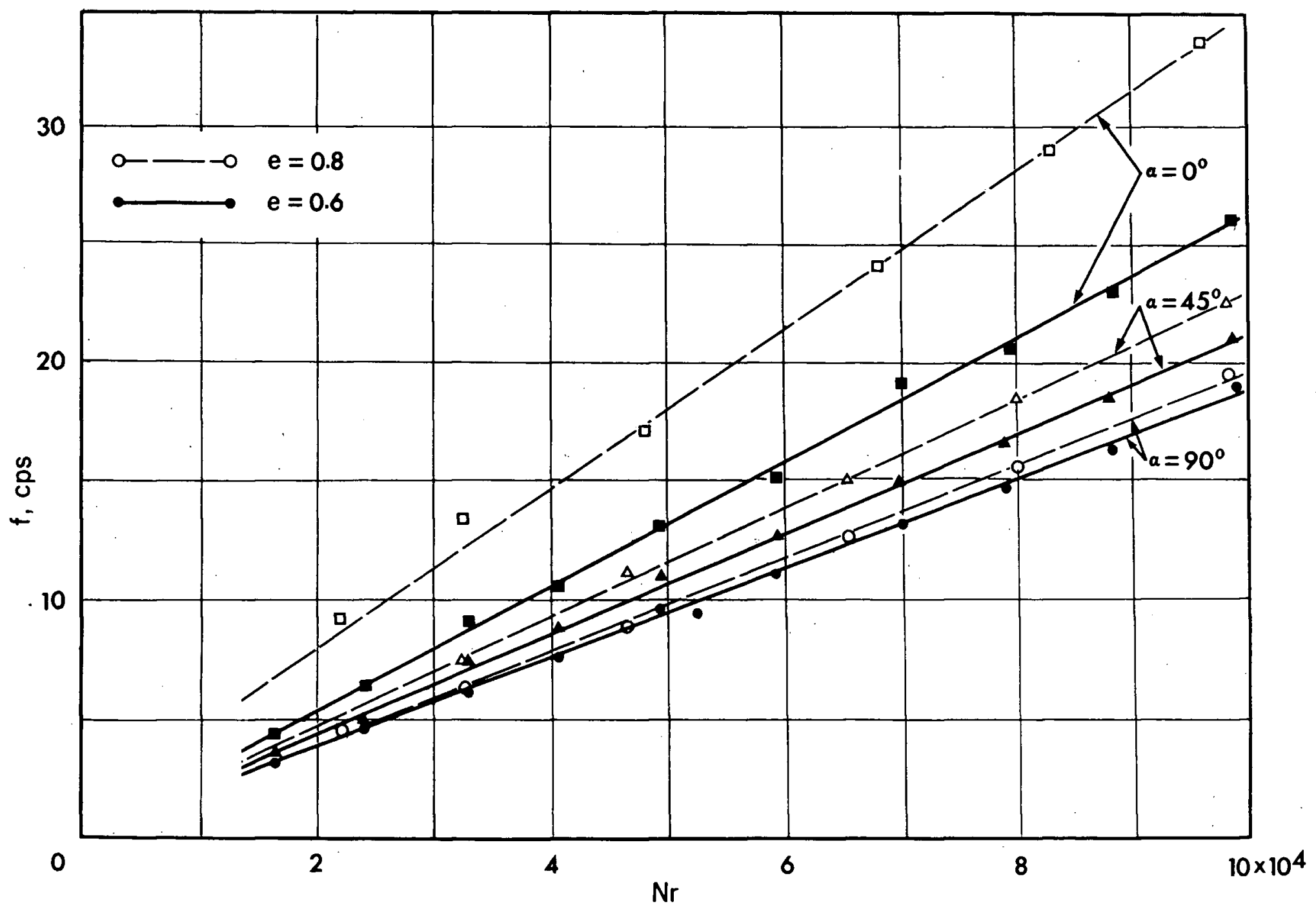

Figure 27... Variation of Stirouhail frequency with Reynolds number 


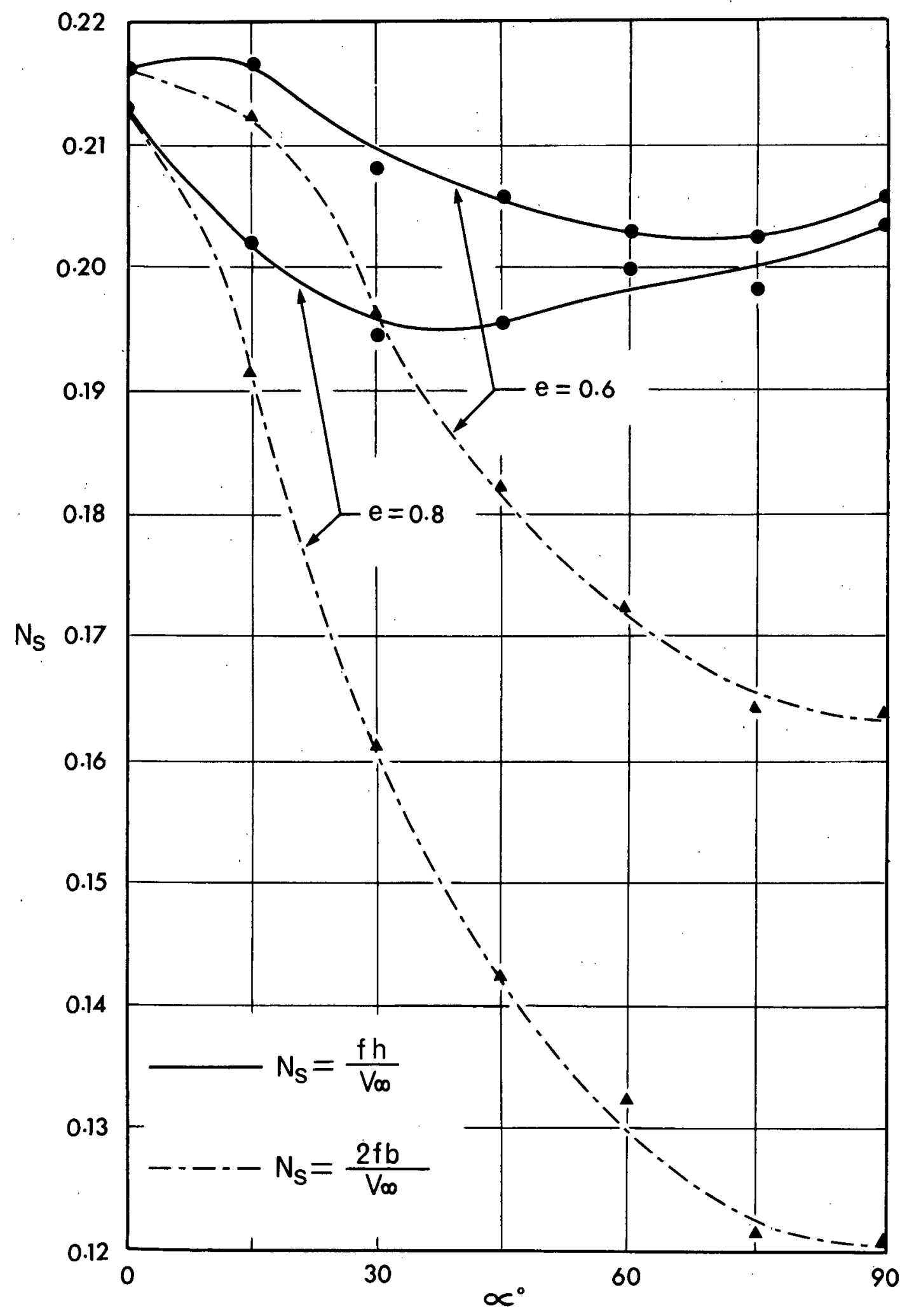

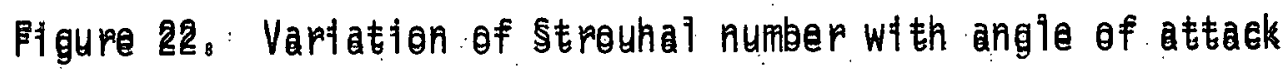


Schramm does not give any details of the wind tunnel test section geometry and the measuring equipment used:

A modulation of the vortex shedding frequency was present at a 11 times. It amounted to as much as $\pm 5 \%$, and may be related, to some. extent, to the amplitude modulation. The frequency:modulation was too small to affect the filter calibration.

\subsection{Fluctuating Pressure Distribution}

For both ellipses, the effect of varying the Reynolds number in the range $3 \times 10^{4}$ to $10^{5}$ was investigated for $\alpha=0,30^{\circ}, 60^{\circ}, 90^{\circ}$. The fluctuating pressure measurements were taken at four taps, two in the laminar and other two in the separated flow regions. The results are plotted in Figures 23,24 . These curves represent the percentage variation about the mean of the pressure coefficient at each of the ports in question.

No significant Reynolds number effect was noticed except for the abrupt increase in the fluctuating pressure coefficient at zero angle of attack and low wind speed. The cause of this phenomenon is not quite clear, but it should be emphasized that it is not associated with any noticeable change in base pressure. This, together, with the Reynolds number at which the behaviour occurred, makes it unlikely to be due to either proximity to the critical Reynolds number or any other change in character of the separation. However, it should also be mentioned that fluctuating signals in this region are very weak and distorted by noise. 


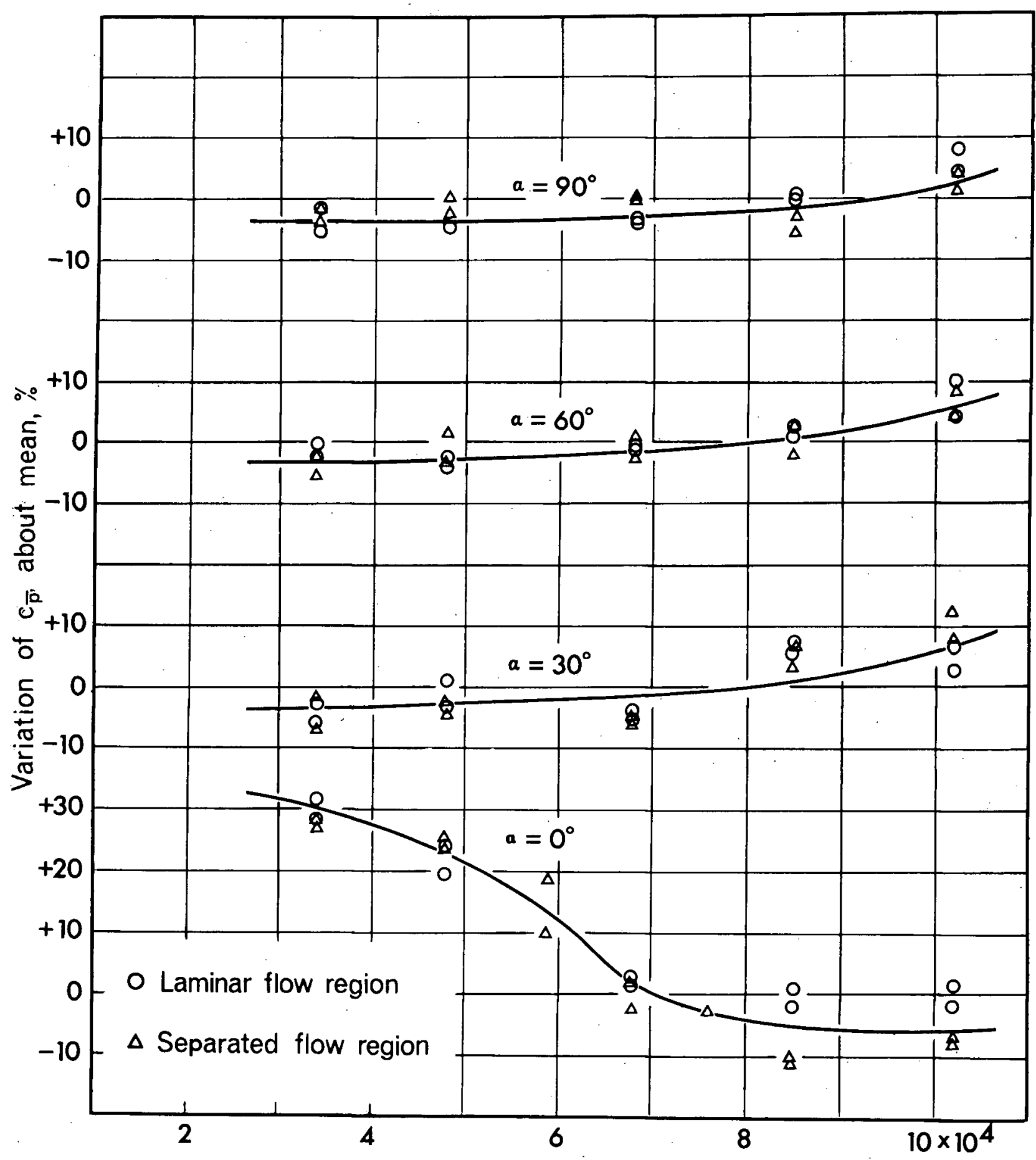

Figure 23, Variation of fluctuating pressure coefficient with Reynolds number, $e=0.6$ 


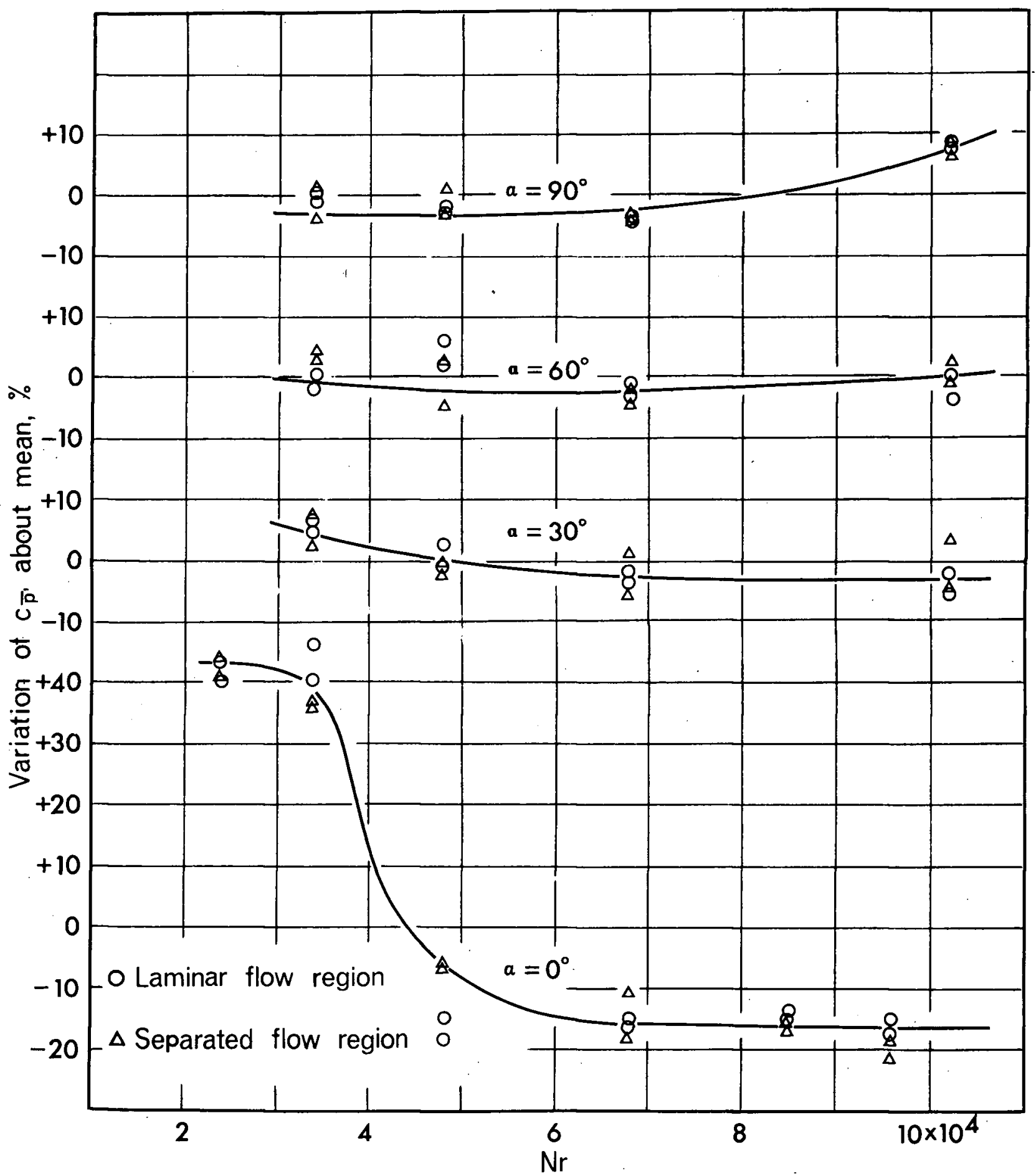

Figure 24. Variation of fluctuating pressure coefficient with Reynolds number, $\mathrm{e}=0.8$ 
In general, the best signals we re obtained at a wind speed around $28 \mathrm{ft} / \mathrm{sec}(\mathrm{Nr} \approx 68,000)$. It was, therefore, decided to carry out fluctuating pressure measurements at this wind speed.

The unsteady pressures on the surface of the two elliptic models were recorded for $\alpha=0,30^{\circ}, 60^{\circ}, 90^{\circ}$ : These results are plotted in Figures 25-28 together with the mean pressure distribution. For comparison the corresponding data for a circular cylinder are presented in Figure 29.

Based on these results the following remarks can be made:

(i) There are two points where the fluctuating pressure tends to vanish. They occupy positions which are approximately $180^{\circ}$ from the stagnation points. One would expect this due to cancellation of pressures which are $180^{\circ}$ out of phase. As shown in the figures this effect is less complete at the rear of the cylinder, probably due to irregularities in the wake.

(ii) The fluctuating pressure increases as the mean pressure decreases and, in general, the variations can be represented by curves following a similar trend.

(iii) The mean pressure increases negatively with angle of attack. The same is true for the unsteady pressure. But, while the mean pressure coefficient approximately doubies in the range $\alpha=0-90^{\circ}$ the corresponding increase in fluctuating pressure coefficient is as high as 10 to 20 times.

(iv) As expected, at zero angle of attack, the fluctuating pressure coefficient for the slender ellipse is considerably less than that for the thicker ellipse, but at $90^{\circ}$ they are practically equal. 


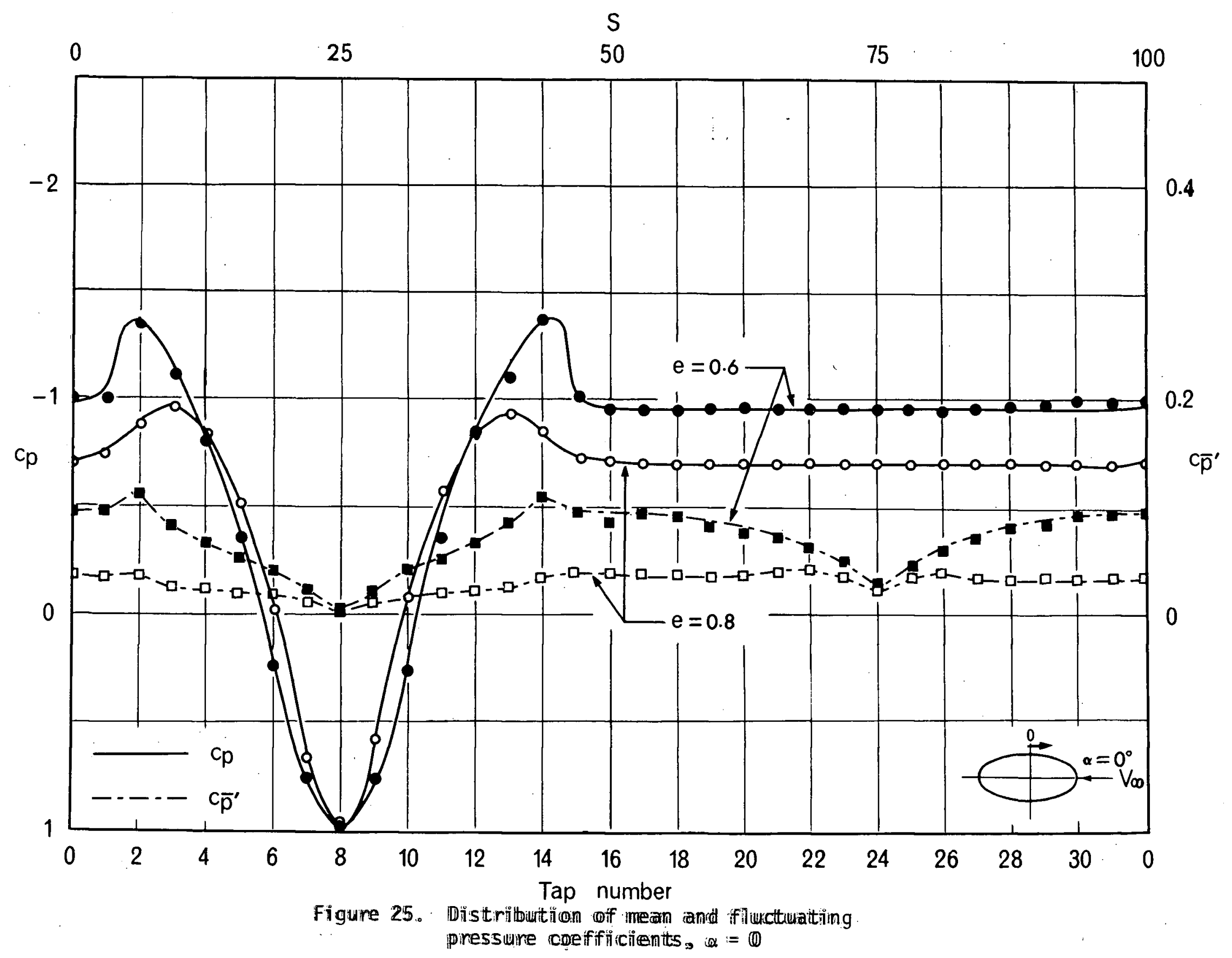




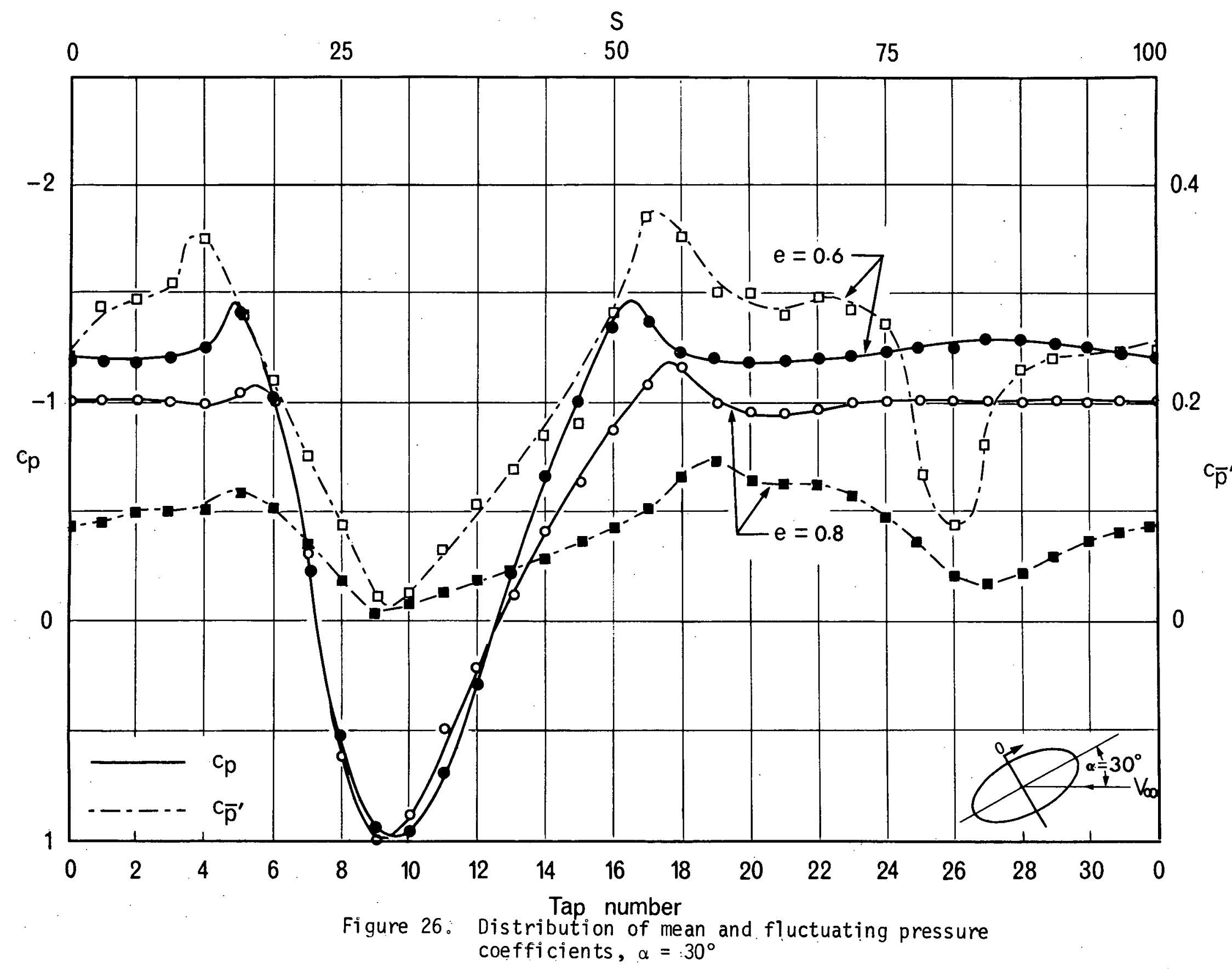




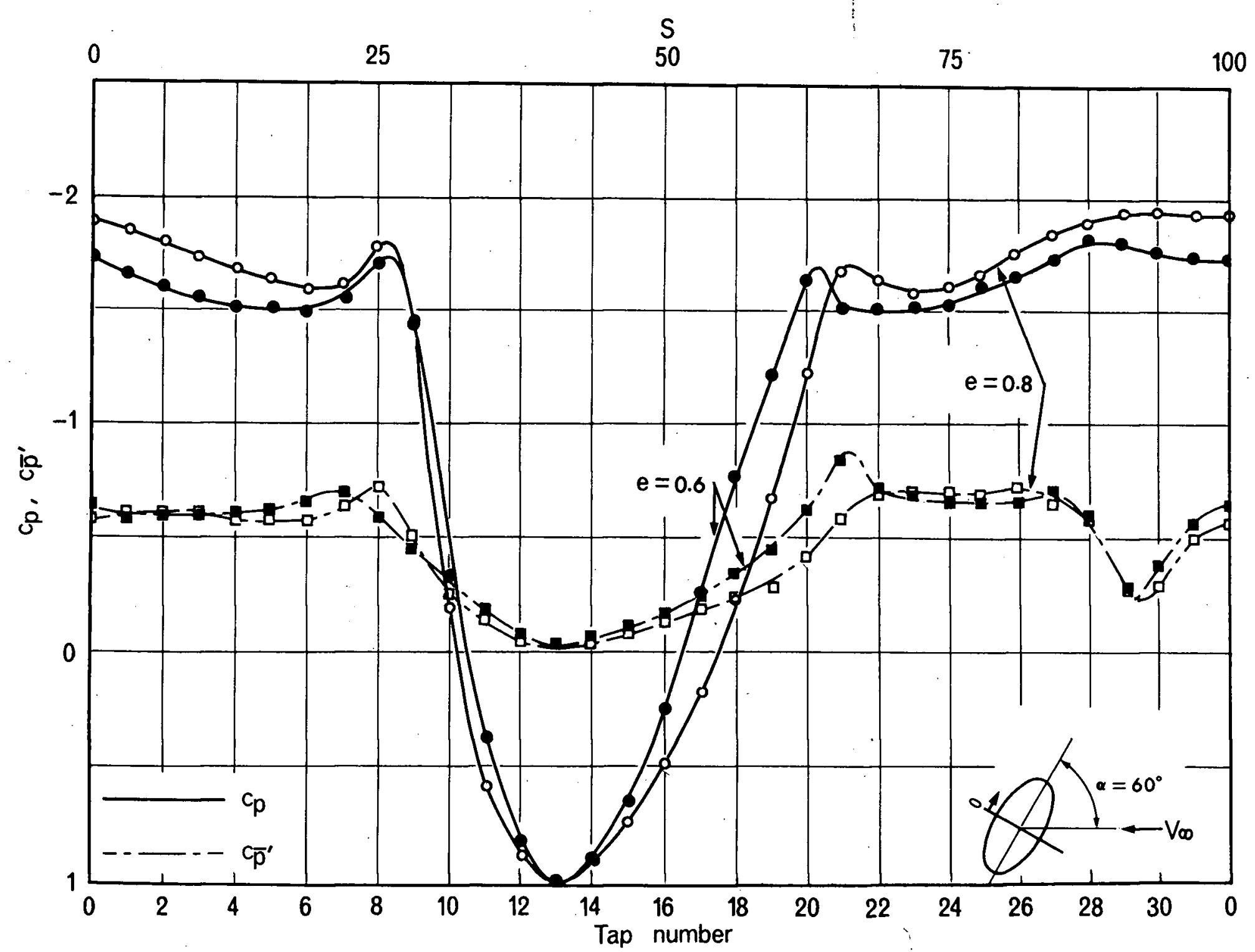

Figure 27: Distribution of mean and fluctuating 


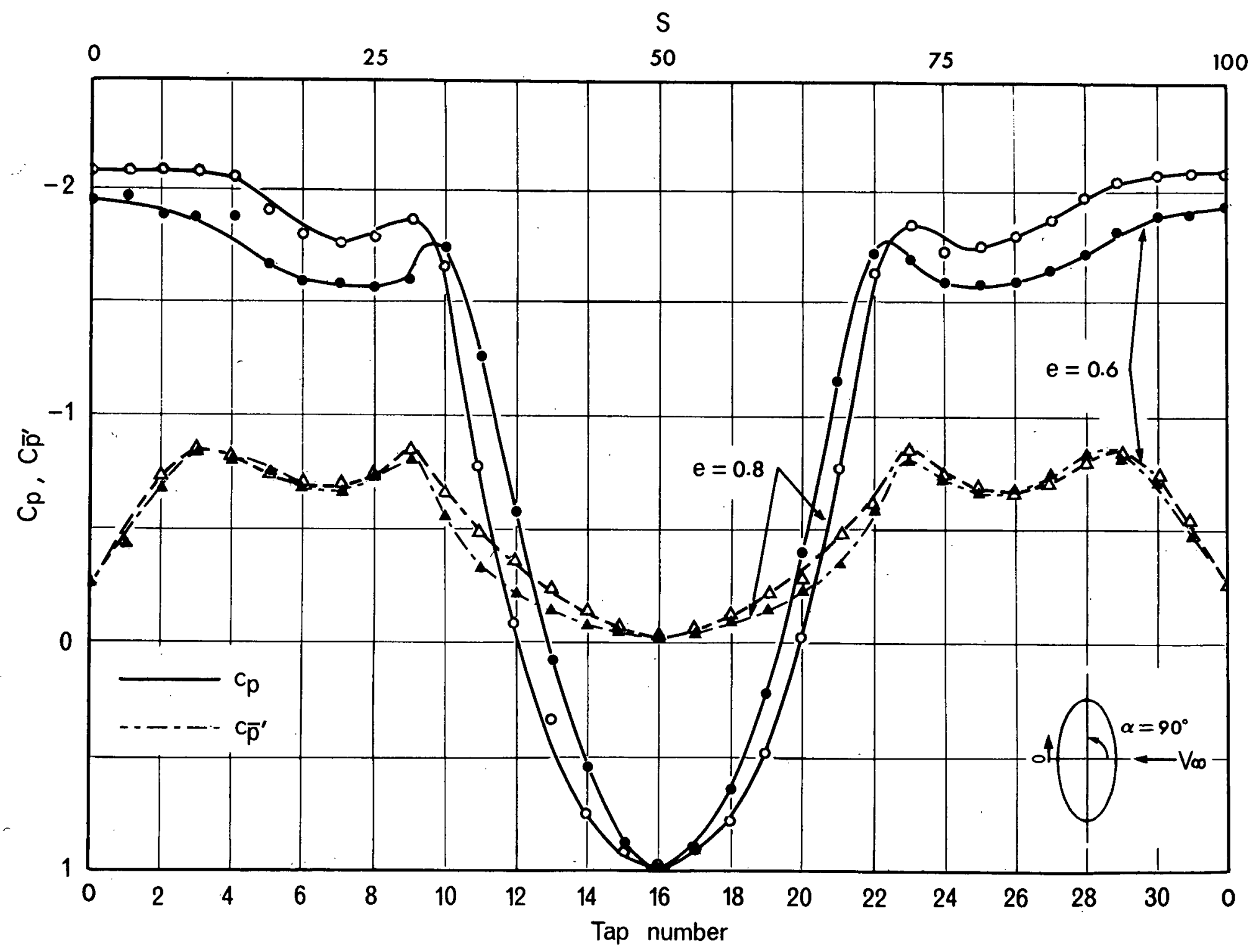

Figure 28: Distribution of meani and fluctuating pressure coefficicients, $\alpha=90^{\circ}$. 


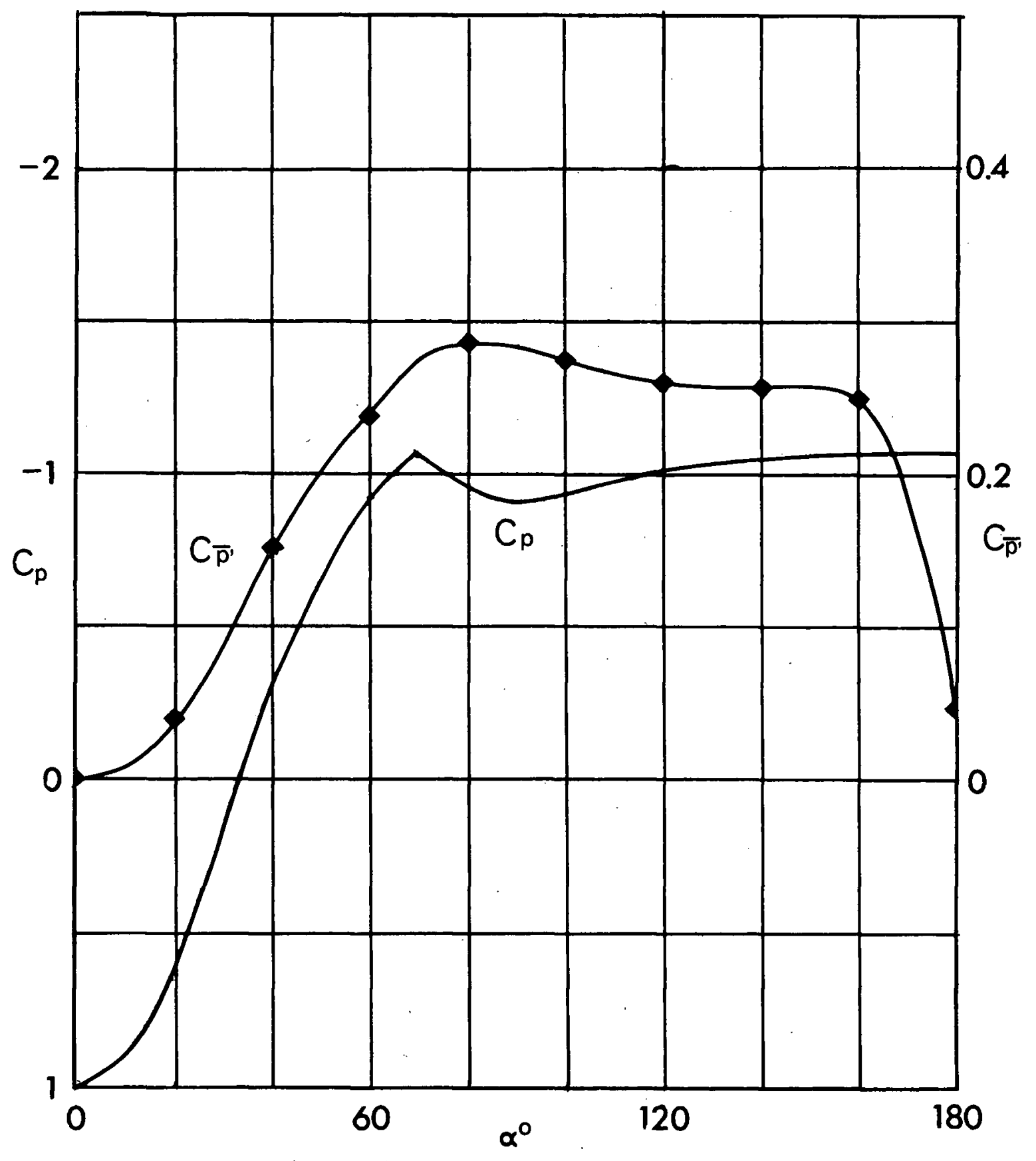

Figure 29. Distribution of mean and fluctuating pressure coefficients on the surface of a circular cylinder, $(e=0)$ 
Variation of the maximum fluctuating pressure coefficient with angle of attack is shown in Figure 30 .

To illustrate the amount of amplitude modulation present in the fluctuating pressure signals, the ratio between the maximum and the average amplitude has been plotted in Figures 37 and 32. The maximum amplitude used is a representative value observed during a two minute period.

The results showed considerable scatter, but the increase in maximum/average ratio towards the rear "stagnation point" was quite distinct. There was also a trend towards a reduction of the ratio in the laminar flow region. As stated by several investigators $10,11,15,16$ the amplitude modulations were found to be in phase around the models.

\subsection{Phase Shift}

Gerrard $^{11}$ was probably the first one to measure phase relation between the fluctuating pressure signals on a circular cylinder. He used two built-in pressure transducers which could be rotated individually and concluded that the pressures were essentially in phase over. one side of the model and $180^{\circ}$ out of phase with that on the other side: Any deviation from this was attributed to the spanwise separation between the pressure taps.

The present experiments confirmed the $180^{\circ}$ phase difference between pressures on two sides of the model. But contrary to Gerrard's observation it indicated substantial phase shift between the signals from the neighbouring pressure taps. The phase measurement was carried out on both elliptic models at $\alpha=0,90^{\circ}$, and the results are plotted 

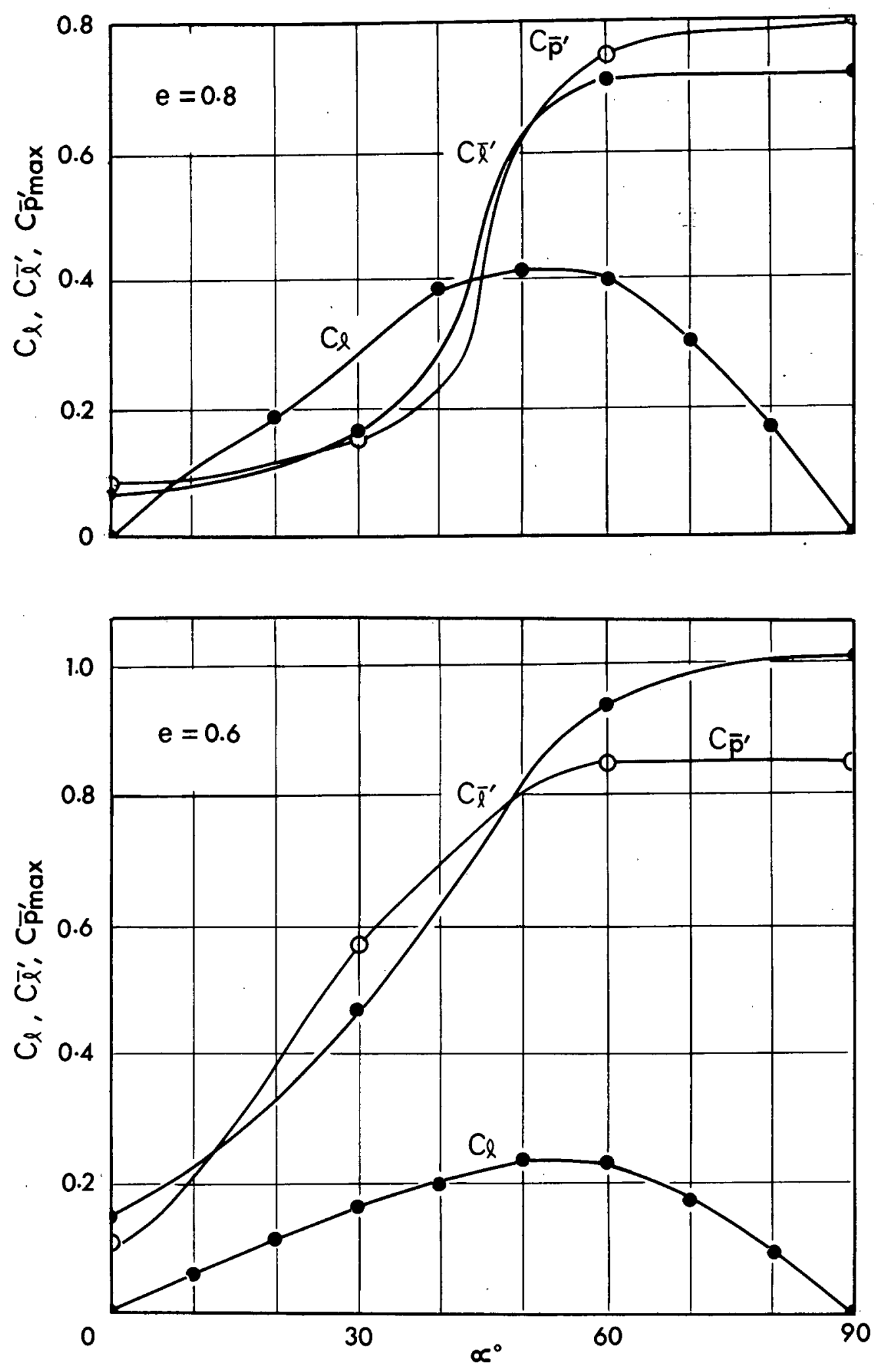

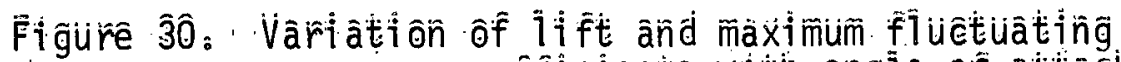

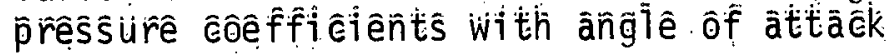




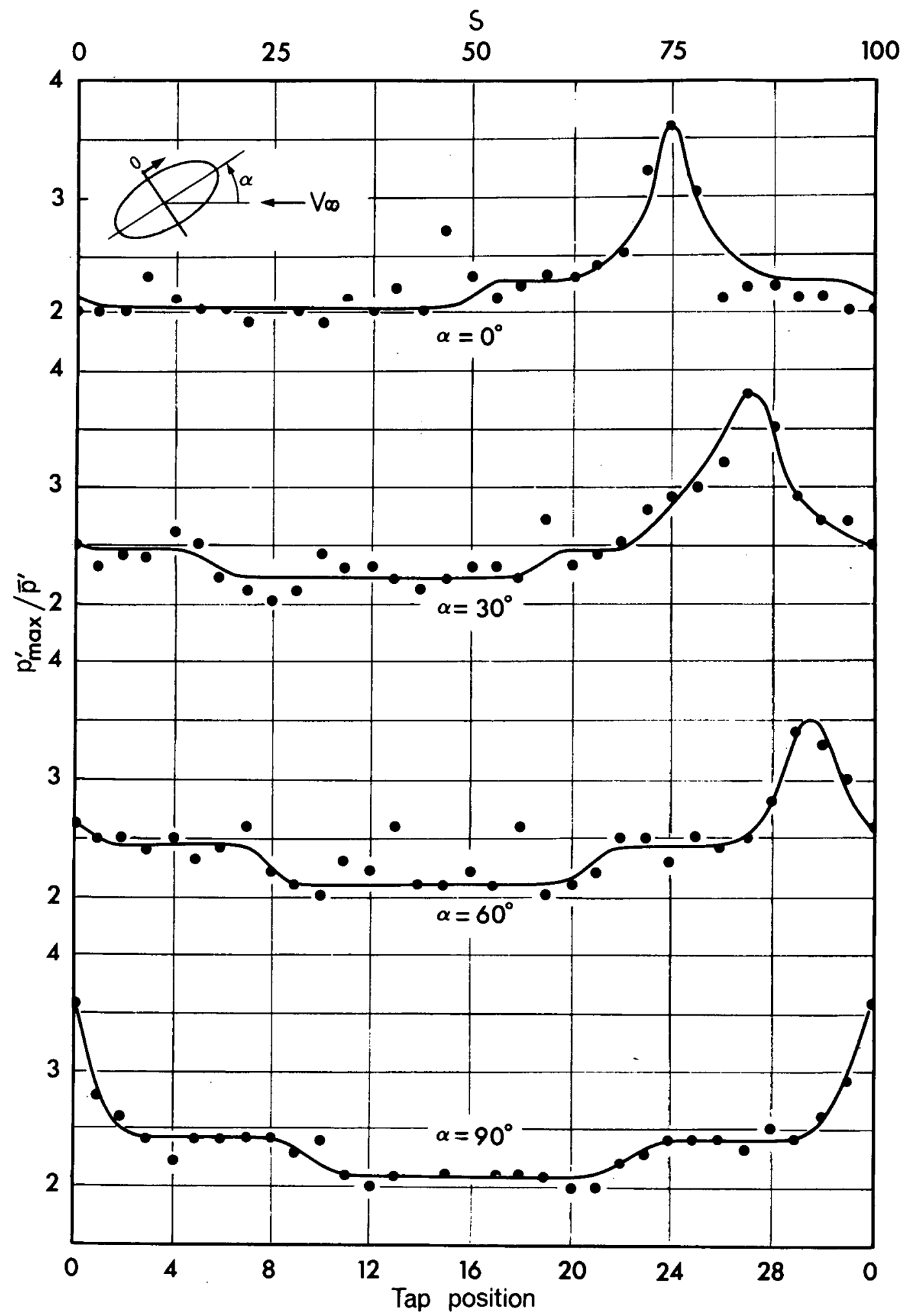

Figure 31. Amplitude modulation of the pressure signals on the surface of the model, $\mathrm{e}=0.6$ 


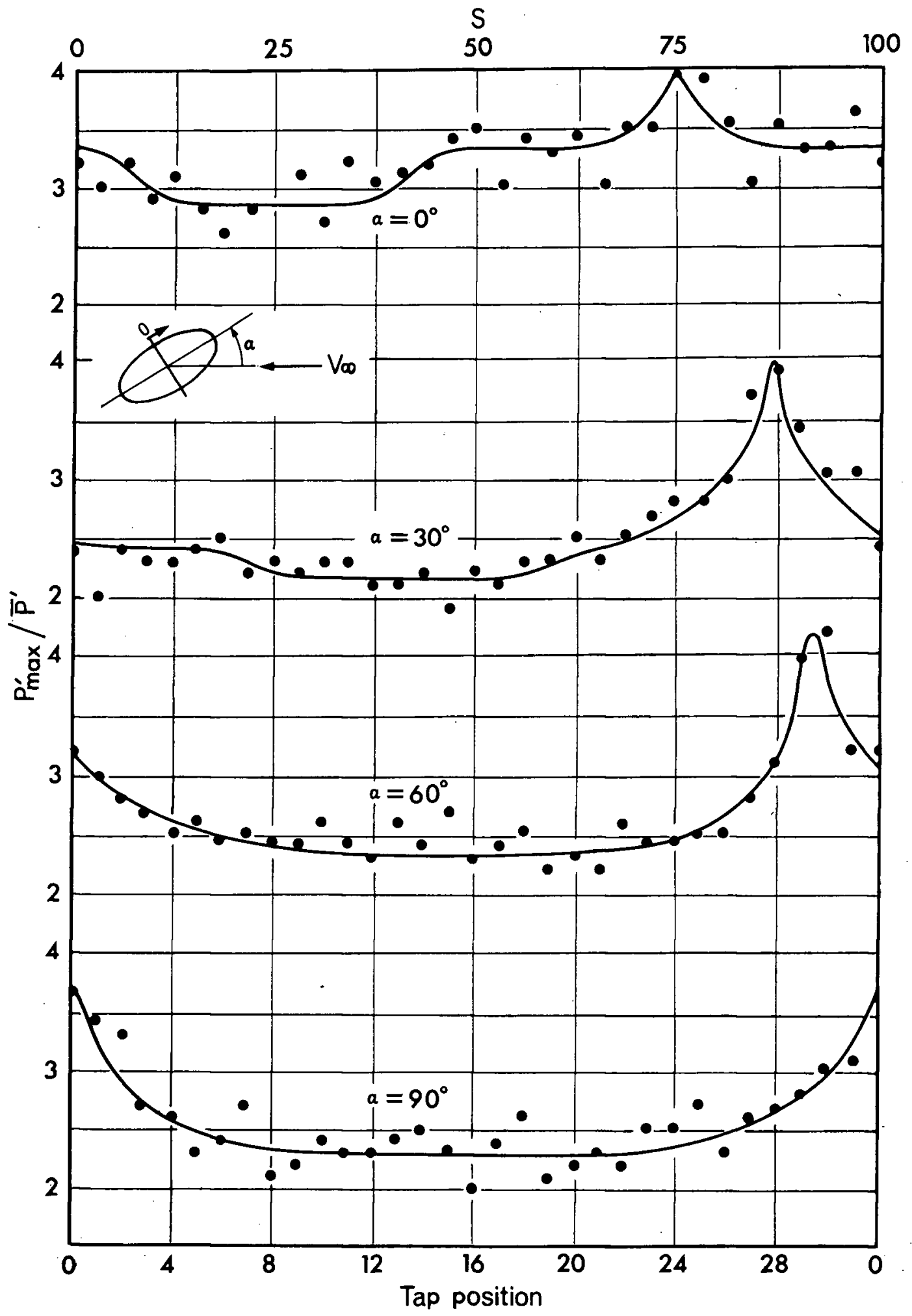

Figure 32. Amplitude modulation of the pressure signals on the surface of the model, $\mathrm{e}=0.8$ 
in Figures 33,34 .

It is apparent that for the models at $\alpha=0$ the signals from upstream and downstream pressure taps are lagging and leading respectively with respect to the reference located at the $90^{\circ}$ position (tap 16 or 32). On the other hand at $\alpha=90^{\circ}$ all signals lag the reference (tap 8 or 24). The phase difference between two pressure signals was found to be as large as $60^{\circ}$.

\subsection{Fluctuating Lift}

The fluctuating lift coefficients for both ellipses at $\alpha=0$, $30^{\circ}, 60^{\circ}$, and $90^{\circ}$ were calculated from the pressure data given earlier.

The results are plotted in Figure 30. For either ellipse the maximum fluctuating lift coefficient $\left.\left[(1.0)_{e}=0.6,(0.72)_{e}=0.8\right)\right]$ was found at $90^{\circ}$ while the minimum value occurred at $\alpha=0$. The fluctuating lift coefficient for a circular cylinder as obtained by McGregor ${ }^{10}$ was 0.6 .

It may be pointed out that the maximum and minimum values given above were obtained without taking phase shift into account. With phase angle, between the pressure signals the corresponding values are modified as shown in Table 2 .

\begin{tabular}{|c|c|c|c|c|}
\hline$e$ & $\alpha$, Deg. & $C_{\bar{\ell}}$ & $\left.C_{\bar{\ell},}\right|_{\text {phase }}$ & \% change in $C_{\bar{\ell}}$ \\
\hline 0.6 & 0 & 0.156 & 0.152 & 2.5 \\
& 90 & 1.007 & 1.001 & 0.5 \\
\hline 0.8 & 0 & 0.064 & 0.060 & 6.0 \\
& 90 & 0.719 & 0.710 & 1.0 \\
\hline
\end{tabular}

Table 2. Effect of phase shift on fluctuating lift coefficient 


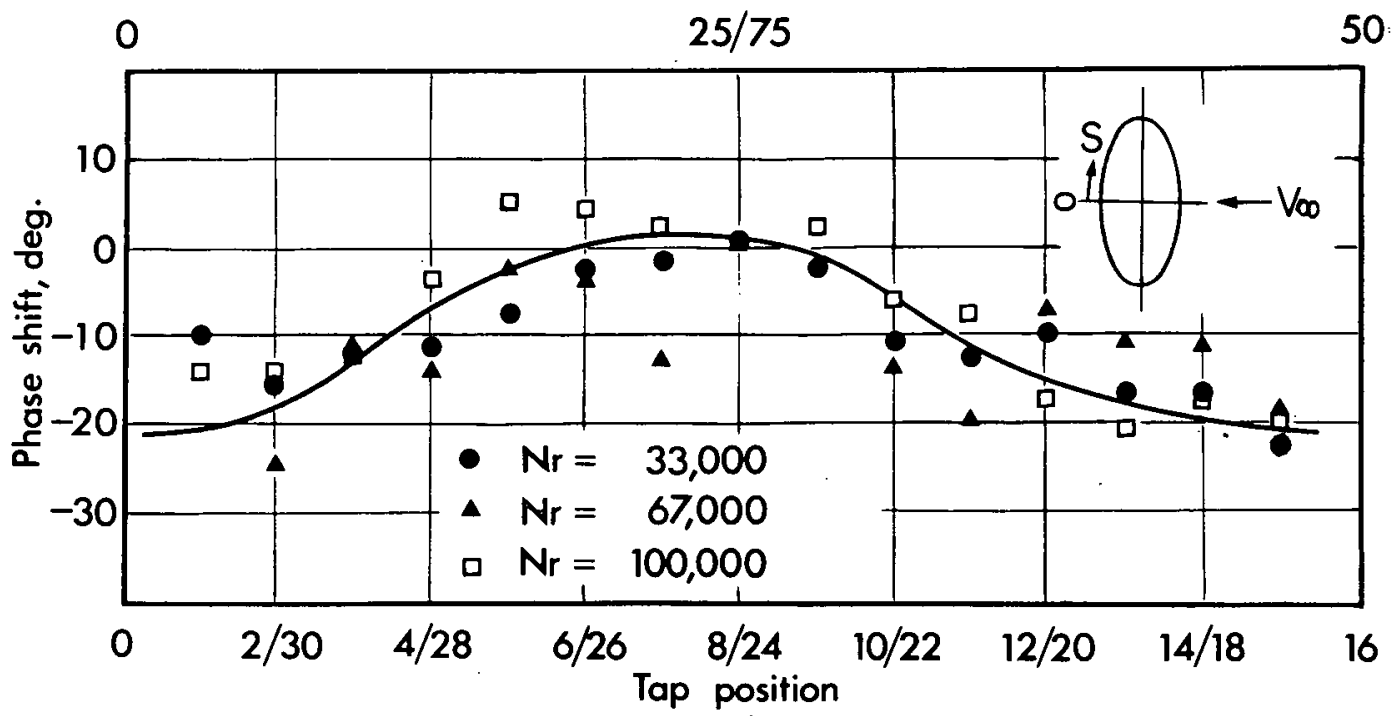

(a)

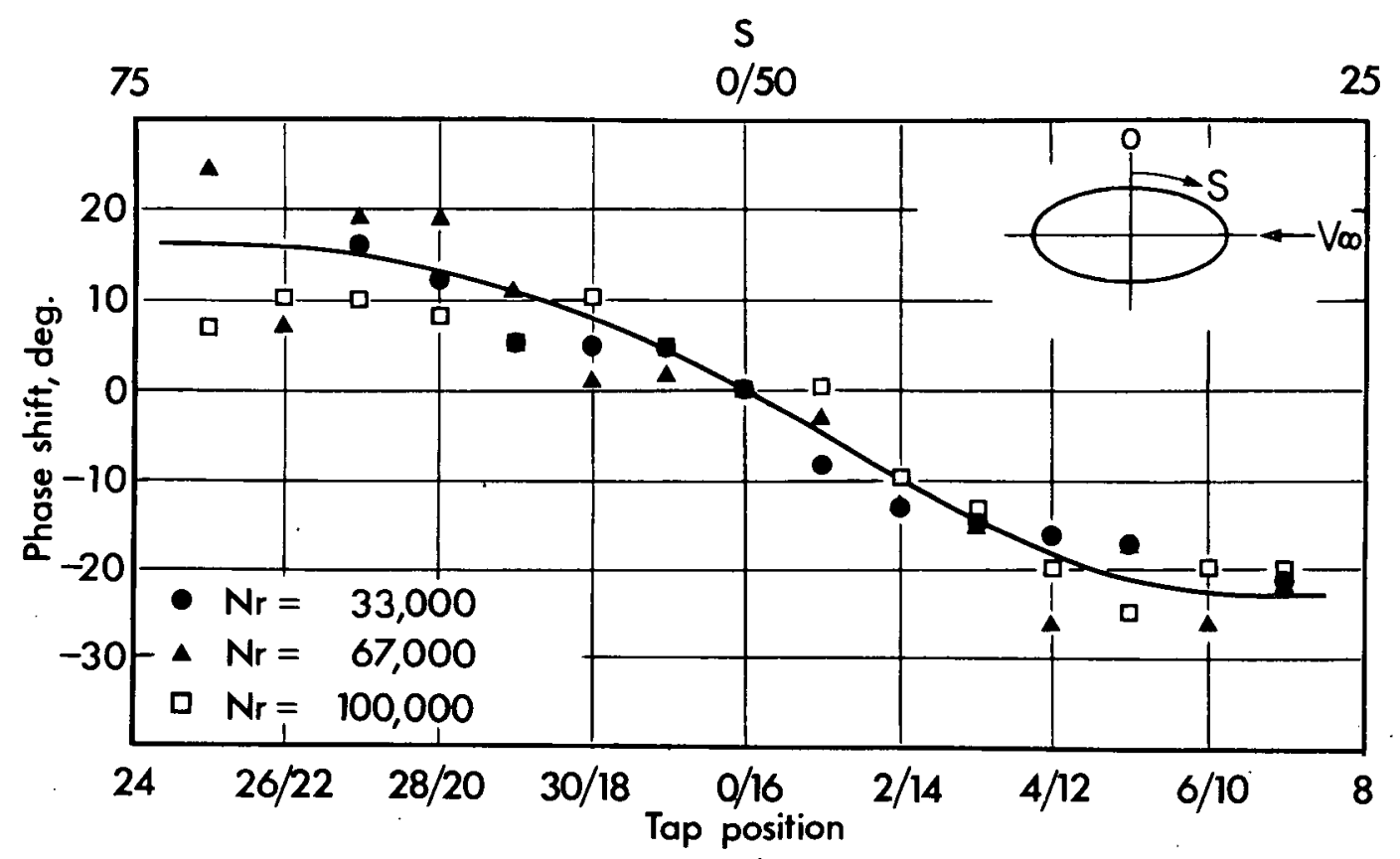

(b)

Figure 33. Phase shift between pressure signals on the surface of the model, a $\equiv 0,6$, $a \equiv 0, \mathrm{g0}^{\circ}$ 


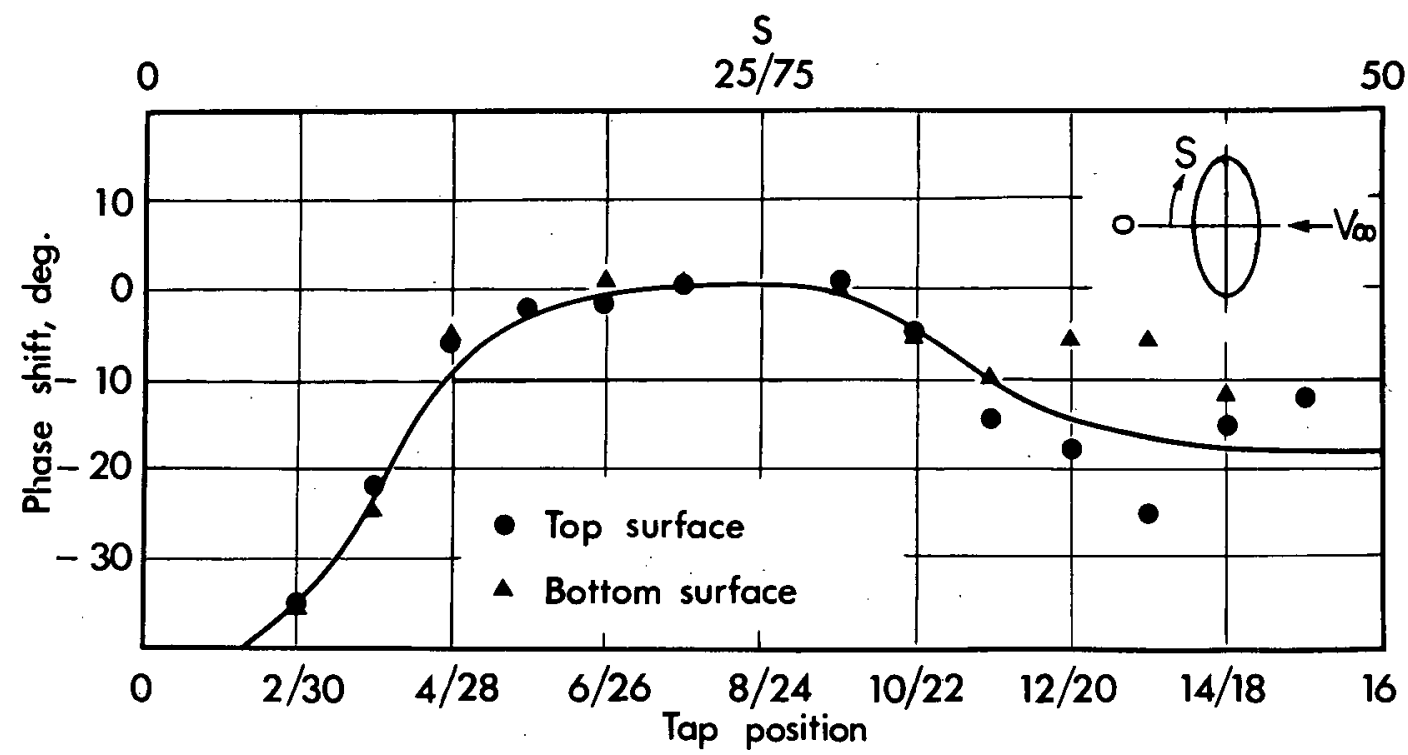

(a)

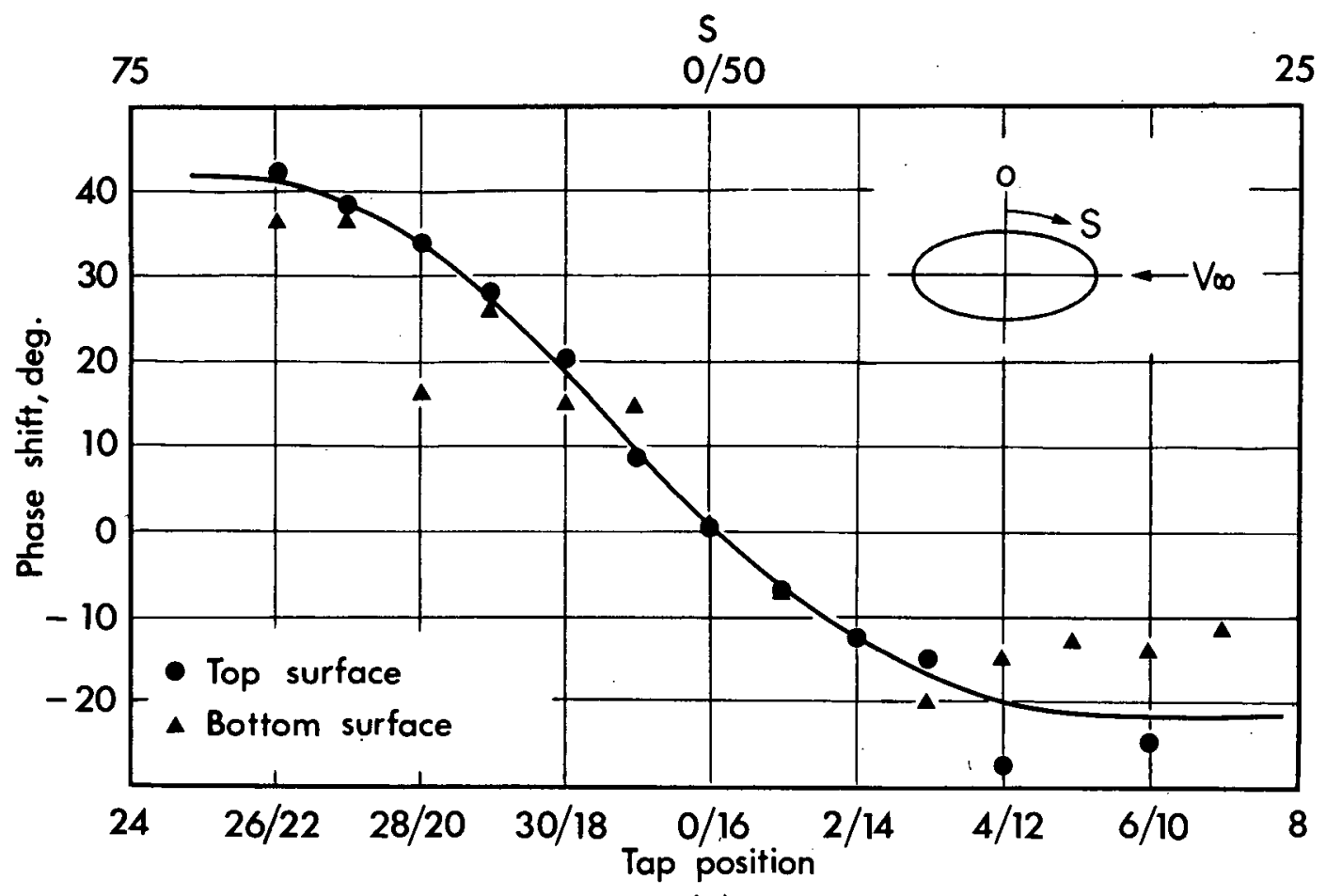

(b)

Figure 34. Phase shift between pressure signals

on the surface of the model, $e=0.8$, $\alpha=0 ; 90^{\circ}$ and $\mathrm{Nr}=67,000$ 
For fluctuating drag and moment coefficients the influence of phase angle may be greater, but no attempt has been made to evaluate these parameters since they are more sensitive to errors in pressure and phase measurements, which occur near the stagnation points:

\subsection{Spanwise Effects}

The validity of assuming two dimensional flow in the present case may be questionable ${ }^{12}$ : Measurements at two spanwise taps (4.5 in. and 9 in. from center section, taps 33 and 34 ) indicated substantial phase difference. Occasionally the phase shift between the signals was observed to remain steady for a short period, but essentially it varied randomly. This, in part, may be attributed to end conditions.

It was not practical with available instrumentation to measure, quantitatively, the time dependent phase shift. However, the presence of spanwise phase difference may not necessarily influence the fluctuating lift too much. The calculations showed a maximum drop of $10 \%$ in fluctuating $1 \mathrm{ift}$ for a phase shift up to $50^{\circ}$ at $\alpha=0$, or up to $30^{\circ}$ at $\alpha=90^{\circ}$. This holds for both ellipses.

Measurements showed the amplitude modulation to be essentially in phase.

\subsection{Wake Geometry}

Typical average amplitude signals across the wake are given in Figure 35a. It was found that after a downstream distance of nearly three major axes the spacings between the vortices remained approximately constant. The wake signals at $\alpha=0$ became too weak 
to be recorded at 20-30 in. downstream. On the other hand more powerful signals at higher angles of attack could be observed down to 45 in. which was the limit for the measuring rig. For $\alpha=30^{\circ}$ and $60^{\circ}$ the wake was slightly unsymmetrical, the vortex from the rearmost separation point being the stronger. Typical decay of pressure amplitude with downstream distance is given in Figure 35b. Average values for the spacing between vortices are given in Table 3 .

\begin{tabular}{|c|c|c|c|c|c|c|c|}
\hline \multirow{2}{*}{$\alpha$} & \multicolumn{3}{|c|}{$e=0.6$} & \multicolumn{3}{c|}{$e=0.8$} & $e=0 *$ \\
\cline { 2 - 8 } & L, In. & W, In. & $W / L$ & L, In. & W, In. & $W / L$ & $W / L$ \\
\hline 0 & 16.00 & 5.0 & 0.31 & 10.00 & 4.3 & 0.42 & 0.32 \\
30 & 19.25 & 5.2 & 0.27 & 16.50 & 5.1 & 0.31 & \\
60 & 21.75 & 5.7 & 0.26 & 20.75 & 5.7 & 0.28 & \\
90 & 22.75 & 5.8 & 0.26 & 22.25 & 5.9 & 0.26 & \\
\hline
\end{tabular}

*Reference 16

Table 3: Spacing of vortices in fully developed wake, $\mathrm{Nr}=70,000$

No quantitative pressure measurements were made in the wake as the probe was not calibrated, but the maximum pressure signals immediately behind the model were estimated to be around 5 times higher than that on the model surface. Within the Range of the Reynolds number investigated $\left(3 \times 10^{4}<\mathrm{N}_{r}<10^{5}\right)$, no significant change in wake geometry occurred. 
6.7 Static Lift, Drag and Moment

The stationary values of lift, drag and moment were calculated from the static pressure results given earlier (Figures 25-28). The variation of static lift coefficient with angle of attack is given in Figure 30. The drag and moment coefficients are plotted in Figure 36. The measured results are uncorrected for wall effects and therefore are somewhat higher than the values corresponding to the unconfined stream.

Approximate corrections to the drag coefficients may be obtained from the expressions given by Whitbread ${ }^{23}$. It amounts to $12 \%$ for both ellipses at $\alpha=90^{\circ}$. At $\alpha=0$ it is $10 \%$ for $e=0.6$ and $8 \%$ for $e=0.8$. As suggested by Whitbread the same corrections may be applied to the lift and moment coefficients (Appendix I). 


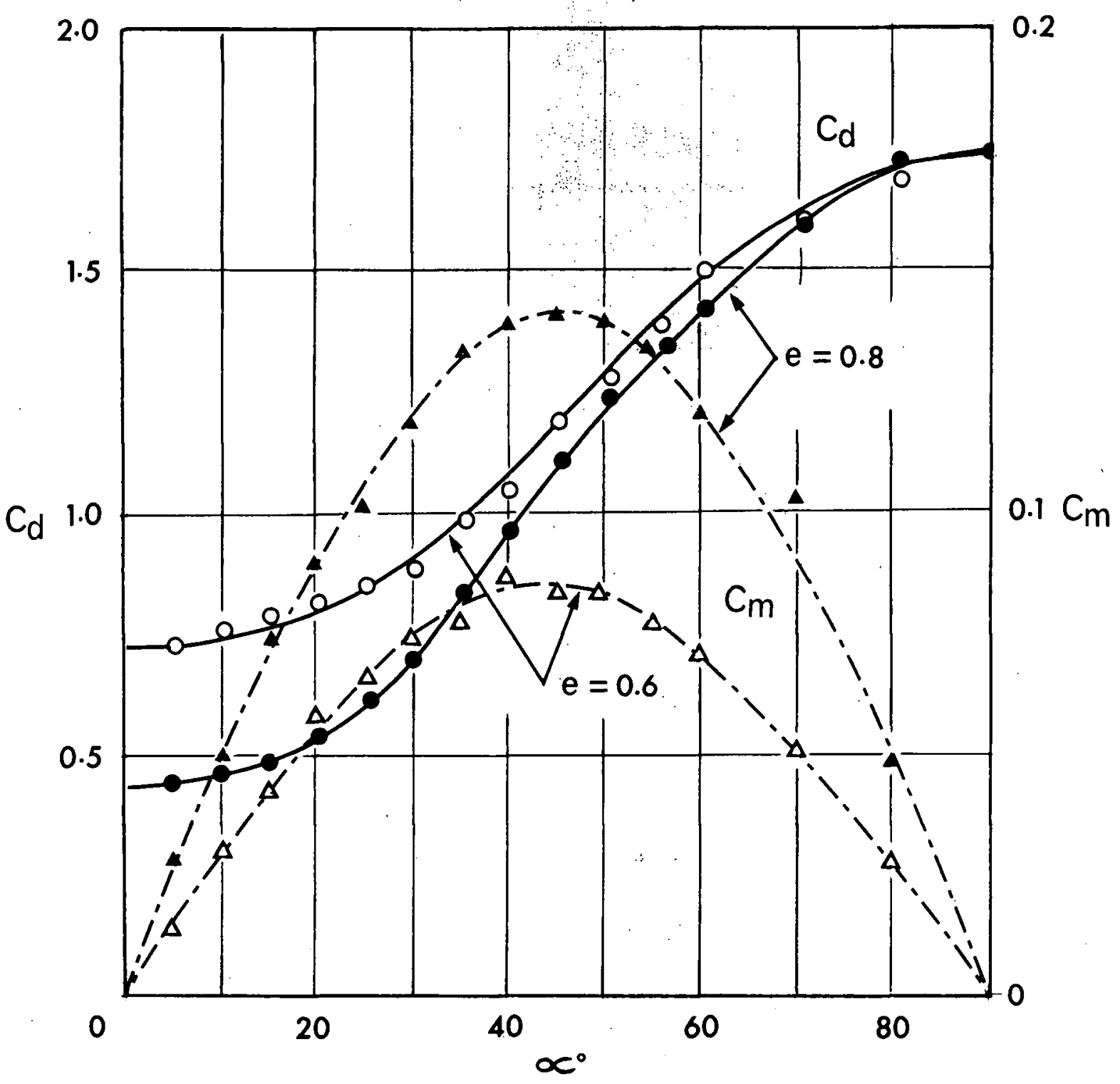

Figure 36. Variation of drag and moment coefficients with angle of attack 


\section{CONCLUDING REMARKS}

Based on the experimental results the following general remarks can be made concerning unsteady aerodynamics of the elliptic cylinders tested:

(i) The Strouhal frequency, which shows slight modulation, increases linearly with wind speed. The variation of Strouhal number with angle of attack is considerably less when based on projected height.

(ii) The fluctuating pressure coefficients tend to increase with angle of attack. In the range $\alpha=0-90^{\circ}, \mathrm{C}_{\mathrm{p}}$, increased from 0.1 to 0.8 for $e=0.6$ and from $0.04^{\max }$. to 0.8 for $e=0.8$. Marked dependency on the Reynolds number appears to be limited to zero angle of attack condition. The pressure signals are always amplitude modulated and the extent of modulation, as expressed by $\frac{p_{\max }^{\prime}}{\bar{p}^{\prime}}$ is of the order $2-4$.

(iii) Pressure signals on the surface of the models have considerable phase differences. At times this can be as high as $60^{\circ}$.

(iv) Similar to the fluctuating pressure coefficients, the lift coefficients are affected by the attitude of the models. The maximum lift coefficients (based on the major axis) were found to be 1.0 and 0.7 for $e=0.6$ and 0.8 respectively. 
The phase shift has only small effect on these values.

(v) The quantitative observations indicate the unsteady flow condition to be far from two dimensional. Based on the observation in (iv) the spanwise variations in phase between the pressure signals is not likely to affect the fluctuating lift substantially.

(vi) The ratio of the transverse to the longitudinal spacing decreases with increasing angle of attack. This was observed to be true for both ellipses but the reduction was more pronounced for the thinner ellipse.

(vii) For $\alpha=0$ the separation points for the boundary layer. as indicated by the static pressure distribution occurs at approximately the same angular position $\left(\approx 75^{\circ}-80^{\circ}\right)$ for both ellipses.* This appears reasonable compared to the experimentally measured value of $\approx 82^{\circ}$. for a circular cylinder.

It may be pointed out that the variation of separation points with eccentricity, and angle of attack, does not seem to be reported in literature. 
A few suggestions concerning the future studies may be appropriate here:

(i) For better appreciation of the transitional effects from circular cylinder to flat plate, further investigations of cylinders with different eccentricity should be undertaken.

(ii) The present technique for phase measurements involves considerable efforts to get meaningful results. Some direct method of obtaining the time average of the phase shift is certainly desirable.

(iii) The Reynolds number effects at zero angle of attack should be studied in detail.

(iv) A study of the three-dimensional nature of the flow should be of interest. A refined phase measuring device might. prove useful in such a study.

(v) The experimental measurements of separation points on cylinders of different eccentricity and its correlation with theory should prove to be a valuable study.

(vi) Of course, the study of aerodynamics and dynamics of the models during self-excited motion would be the logical extension of this work. 


\section{BIBL IOGRAPHY}

1. Strouha 1, V., "Über eine Besondere Art der. Tonerregung," Wied. Ann. Physik u。Chem。, Neue Folge, Vol。:V, 1878, pp。216-251.

2. Kárman, Th., Von, "Flussigkeit u. Luftwiderstand," Phys. Z., Vol. 13, 1917, p.49.

3. Marris, A.W.; "A Review on Vortex Streets, Periodic Wakes, and Induced Vibration Phenomena;" Jo' Basic Engng., Vo1. 86, 1964, pp. 185-196.

4. Grove; A.S., Shair; F.H.; Petersen, E.E. and Acrivos, A., "An Experimental Investigation of the Steady Separated Fiow Past a Circular Cylinder," J. Fluid Mech., Vol. 19, part 1, 1964, pp. 60-80.

5. Bishop, R.E.D. and Hassan, A.Y., "The Lift and Drag Forces on a Circular Cylinder in a Flowing Flutd," Proc. Roy. Soc., Series $A$, Vol. 277, 1964, pp. 32-50.

6. Modi, V.J. and Heine, W., "On the Pressure Fluctuations and Wake Geometry Associated with Several Bluff Bodies," Proc. 15th Japan Nat. Cong. App1. Mech., Japan Soc. Mech. Engrs. T965, pp. 7-18.

7. Bishop, R.E.D: and Hassan, A.Y.,. "The Lift and Drag Forces on a Circular Cylinder Osclliating in a Flowing Fluid," Proc. Roy. Soc., Series A; Vol. 277;, 1964, pp. 51-75.

8. Ferguson, N. and Parkinson, G.V., "Surface and Wake Flow Phenomena of the Vortex-Excited Osclllation of a.Circular Cylinder," ASME VIbration Conference, Paper 67-Vtbr.-31, 1967.

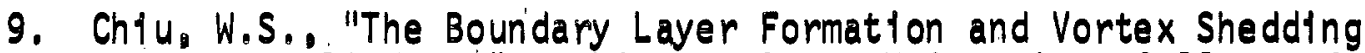
on Yawed. Cylinders," Washington State University, College of Engng; , Bulietin 299, 1966.

10. McGregor, D.M., "An Experimental Investigation of the Oscillating Pressures on a Circular Cylinder in a Fluid Stream," University of Toronto, Inst1tute of Aerophys 1cs, Tech. Note 14, $195 \%$.

11. Gerrard, J.H..."An Experimental Investigation of the Oscillating Pressures on a Circular Cylinder Shedding Turbulent Vortices," Journal of Flutd.Mechanics; Vol. 11!; 1961, pp. 244-256.

12. Prendergast, $V_{\text {. }}$, "Measurement of Two-Point Correlations of the Surface Pressure on a Circular Cylinder," University of Toronto; Institute of Aerophysics, Tech. Note 23, 1958. 
13. Keefe, R.T., "An Investigation of the Fluctuating Forces Acting on a Stationary Circular Cylinder in a Subsonic Stream, and of the Associated Sound Field," University of Toronto, Institute of Aerophysics, Report 76, 196T.

14. Molineux, W.G., "Measurement of the Aerodynamic Forces on Oscillating Airfoils," AGARD Report. 35, 1956.

15. Heine, W.; "On the Experimental Investigation of Vortex Excited Pressure Fluctuations," University of British Columbia, M.A.Sc. Thesis, 1964 .

16. Ferguson, N., "The Measurement of Wake and Surface Effects in the Subcritical Flow Past a Circular Cylinder at Rest and in Vortex Excited Oscilitations," "University of British Columbia, M.A.Sc. Thes is, 1965.

17. Schramm, $W_{0}:$, Wi rbelfrequenzmessungen an umstromten Bauteilen," IfL-Mitt., Vol. 5, 1966, pp. 308-318.

18. Parkinson, G.V., "Aeroelastic Galloping in One Degree of Freedom," Proc. First Int. Conf. on Wind Effects on B1dgs. and Structs., NPL., London, Vol. II, 1965, pp. 581-609.

19. Parkinson, G.V. and Modi, V.J., "Recent Research on Wind Effects on Bluff Two-Dimensional Bodies," Int. Research Seminar: Wind Effects on B7dgs: and Structs., NRC, Ottawa, 1967.

20. Morse, P.M., "Vibration and Sound," McGraw-Hi11, New York, 1948, pp. 233-265.

21. Bryer, D.W., Walshe, D.E. and Garner, H.C., "Pressure Probes Selected for Three-Dimensional :Flow Measurement," Aeronautical Research Council, R. and M. No. 3037, 1958.

22. Cheng, S., "An Experimental Investigation of the Autorotation of a Flat Plate, "University of British Columbia, M.A.Sc. Thesis, 1966 .

23. Whitbread, R.E., "Model Simulation of Wind Effects on Structures," Proc. First Int. Confo on Wind Effects on BTdgs. and Structs., NPL, London, Vol. 2, T965, pp. 581-610.

24. Pankhurst, R.C. and Holde r; 'D.W.; "Wtnd-Tunnel Technique," Pitman \& Sons Ltd., London, 1952, chapter 8.

25. Maske11, E.C., "A Theory of the Blockage Effects on Bluff Bodies and Stalled Wings in a Closed Wind Tunnel," RAE Report No. Aero 2685, Nov. 1963. 
26. Schlichting, H., "Boundary - Layer Theory," McGraw-Hi11, New York, 1968, pp. $21,202-206,475$.

27. Meksyn; D.; "New Methods in Laminar Boundary - Layer Theory," Pergamon Press, London, 1961, chapter 71.

28. Görtler, H., "A New Series for the Calculation of Steady Laminar Boundary Layer Flows," Journal of Mathematics and Mechanics, Vo1. 6, No. 1, 1957, pp. 1-66. 


\section{APPENDIX I}

REMARKS CONCERNING WIND TUNNEL WALL CORRECTIONS

There is considerable information available for prediction of equivalent free flight results from wind tunnel measurements as far as steady flow is concerned. Unfortunately, the same cannot be said for unsteady flow around a body.

As indicated by Pankhurst and Holder 24 the interference from wind tunnel walls during steady flow conditions may be subdivided into:

(i) Solid blockage

(ii) Wake brockage

(iii) Lift effect

(iv). Interference due to static pressure gradient

(v) Wall boundary-layer interference on model spanning a closed. tunnel

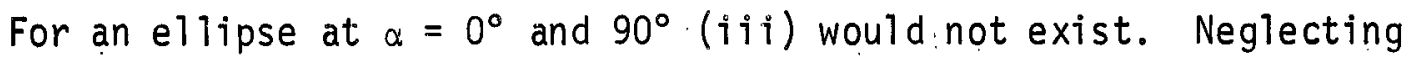
(iv) and (v) gives a correction to wind speed as follows:

$$
\left.v_{\text {corr. }}=v\left[k \cdot \lambda\left(\frac{h}{w_{t}}\right)+n c_{d} \frac{h}{w_{t}}\right)\right]
$$

where

$$
\begin{aligned}
& k=\text { constant } \\
& \lambda=\text { shape factor } \\
& n=\text { wake blockage factor } \\
& w_{t}=\text { tunnel width }
\end{aligned}
$$


This expression gives a correction similar to the one obtained using Whitbread's ${ }^{23}$ equation, which is a particular form of a more general expression given by Maske $11^{25}$.

For fluctuating pressures apparently no corrections are available, probably because the situation is considerably more complex. In addition to blockage influencing both fluctuating pressure and vortex frequency, it is likely. that the boundaries will impose a velocity field due to the wake vortices. Moreover, the periodic nature of the flow may lead to an expression for wall correction which is also periodical. 
APPENDIX II

LOCATION OF SEPARATION POINTS ON ELLIPTIC CYLINDERS

The available information on the laminar boundary layer separation on bluff bodies is somewhat scarce and unreliable. The bulk of the information is obtained analytically using boundary layer separation criteria with external flow distribution assumed potential.

This information is summarised in the following chart. The positions of minimum pressure as given by the present set of experiments are also included. 


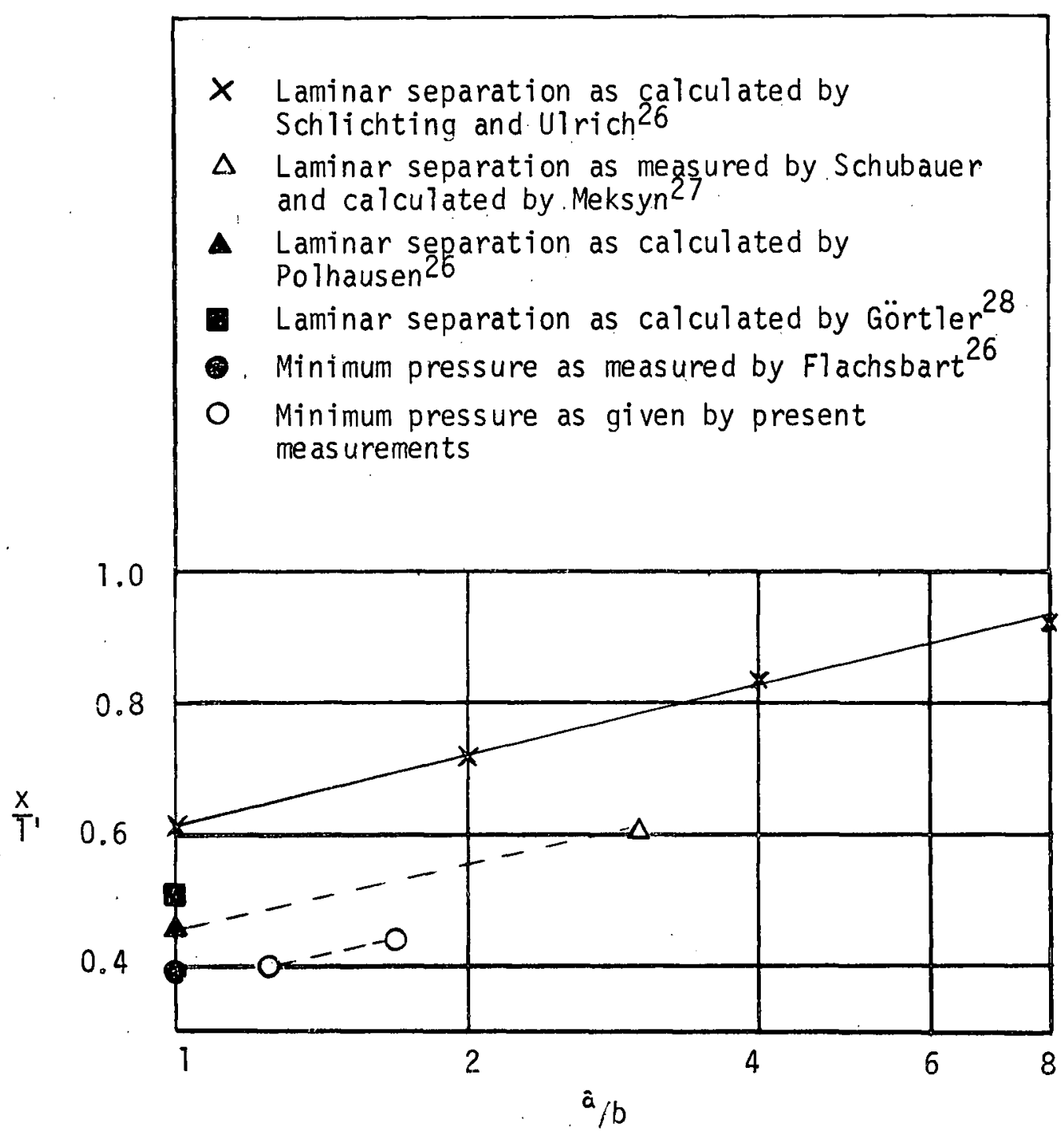

Dependence of points of separation and minimum pressure on bluffness, $\alpha=0$; $x=$ distance along circumference, $l^{\prime}=$ semi ci rcumference. 
........Ah! voilà quatre-vingts volumes de recueils d'une académie des sciences! s'écria Martin. Il se peut qu'il y ait là du bon. -- Il y en aurait, dit Pococurante, si un seul des auteurs de ces fatras avait inventé seulement 1 'art de faire des épingles; mais il n'y a, dans tous ces livres, que de vains systèmes, et pas une seule chose utile.

VOLTAIRE 